Article

\title{
Bimetallic Aluminum 5,6-Dihydro-7,7-dimethyl quinolin-8-olates as Pro-Initiators for the ROP of $\varepsilon-C L$; Probing the Nuclearity of the Active Initiator
}

\author{
Qiurui Zhang ${ }^{1,2}$, Wenjuan Zhang ${ }^{1,2, *}$, Gregory A. Solan ${ }^{2,3, *}$, Tongling Liang ${ }^{2}$ \\ and Wen-Hua Sun $2, *$ id \\ 1 Beijing Key Laboratory of Clothing Materials R\&D and Assessment, Beijing Engineering Research Center \\ of Textile Nanofiber, School of Materials Science and Engineering, Beijing Institute of Fashion Technology, \\ Beijing 100029, China; zhangqiurui@iccas.ac.cn \\ 2 Key Laboratory of Engineering Plastics and Beijing National Laboratory for Molecular Science, \\ Institute of Chemistry, Chinese Academy of Sciences, Beijing 100190, China; 1t1@iccas.ac.cn \\ 3 Department of Chemistry, University of Leicester, University Road, Leicester LE1 7RH, UK \\ * Correspondence: zhangwj@bift.edu.cn (W.Z.); gas8@leicester.ac.uk (G.A.S.); whsun@iccas.ac.cn (W.-H.S.); \\ Tel.: +86-10-6255-7955 (W.-H.S.)
}

Received: 24 June 2018; Accepted: 10 July 2018; Published: 12 July 2018

\begin{abstract}
Six examples of aluminum 5,6-dihydro-7,7-dimethylquinolin-8-olates, [\{2-R ${ }^{1}-7,7-\mathrm{Me}_{2}-8$ $\left.\left.-\mathrm{R}^{2} \mathrm{C}_{9} \mathrm{H}_{6} \mathrm{~N}-8-\mathrm{O}\right\} \mathrm{AlR}^{3}{ }_{2}\right]_{2}\left(\mathrm{R}^{1}=\mathrm{R}^{2}=\mathrm{H}, \mathrm{R}^{3}=\mathrm{Me} \mathrm{C} 1 ; \mathrm{R}^{1}=\mathrm{R}^{2}=\mathrm{H}, \mathrm{R}^{3}=\right.$ Et C2; $\mathrm{R}^{1}=\mathrm{R}^{2}=\mathrm{H}, \mathrm{R}^{3}=i-\mathrm{Bu}$ C3; $\mathrm{R}^{1}=\mathrm{Cl}, \mathrm{R}^{2}=\mathrm{H}, \mathrm{R}^{3}=$ Me C4; $\mathrm{R}^{1}=\mathrm{H}, \mathrm{R}^{2}=\mathrm{R}^{3}=$ Me C5; $\mathrm{R}^{1}=\mathrm{Cl}, \mathrm{R}^{2}=\mathrm{R}^{3}=$ Me C6), have been prepared by treating the corresponding pro-ligand (L1-L4) with either $\mathrm{AlMe}_{3}, \mathrm{AlEt}_{3}$ or $\mathrm{Al}(i-\mathrm{Bu})_{3}$. All complexes have been characterized by ${ }^{1} \mathrm{H}$ and ${ }^{13} \mathrm{C}$ NMR spectroscopy and in the case of $\mathbf{C} \mathbf{1}$ and C4 by single crystal X-ray diffraction; dimeric species are a feature of their molecular structures. In the presence of $\mathrm{PhCH}_{2} \mathrm{OH}(\mathrm{BnOH}), \mathbf{C 1}-\mathbf{C 6}$ displayed good control and efficiency for the ROP of $\varepsilon$-CL with almost $100 \%$ conversion achievable in $10 \mathrm{~min}$ at $90{ }^{\circ} \mathrm{C}$; the chloro-substituted $\mathrm{C} 4$ and C6 notably exhibited the lowest activity of the series. However, in the absence of $\mathrm{BnOH}, \mathrm{C1}$ showed only low activity with 15\% conversion achieved in 30 min forming a linear polymer capped with either a methyl or a L1 group. By contrast, when one or more equivalents of $\mathrm{BnOH}$ was employed in combination with $\mathbf{C 1}$, the resulting catalyst was not only more active but gave linear polymers capped with $\mathrm{BnO}$ end-groups. By using ${ }^{1} \mathrm{H}$ and ${ }^{27} \mathrm{Al} \mathrm{NMR}$ spectroscopy to monitor solutions of $\mathrm{C1}, \mathrm{C1} / \mathrm{BnOH}$ and $\mathrm{C1} / \mathrm{BnOH} / 10 \varepsilon-\mathrm{CL}$ over a range of temperatures, some support for a monomeric species being the active initiator at the operational temperature is presented.
\end{abstract}

Keywords: aluminum complexes; ring opening polymerization; $\varepsilon$-caprolactone; bidentate; reaction mechanism

\section{Introduction}

The past 10 years or so have seen some rapid progress in the synthesis of biodegradable polymers and in particular, aliphatic polyesters such as polylactides (PLA) and poly( $\varepsilon$-caprolactone) (PCL). These developments can be attributed, in a large measure, to the good biodegradability and biocompatibility properties of these materials as well as to their ease of preparation [1-4]. Typically, such polyesters can be prepared by the ring-opening polymerization (ROP) of cyclic esters catalyzed by metal complexes such as those based on $\mathrm{Al}, \mathrm{Ca}$, Sn and rare earth metals. In addition, immobilized catalysts have been considered for the improvement of the mechanical properties of PCLs [5]. Several review articles have documented advances in catalyst design for the ROP of cyclic esters [6,7]. Among the numerous reports, aluminum complexes bearing multidentate ligands such as Salen-Al and Salan-Al have 
attracted much attention due to their relatively high Lewis acidity, good controllability, as well as their decent selectivity towards the ROP of rac-lactide ( $r a c-L A)$ and $\varepsilon$-caprolactone $(\varepsilon-C L)$ [8-11]. By way of contrast, there are still relatively few examples of bidentate $\mathrm{N}^{\wedge} \mathrm{O}$-type aluminum complexes that have been used effectively for the ROP of cyclic esters.

With regard to $\mathrm{N}^{\wedge} \mathrm{O}$-aluminum complexes containing six-membered chelate rings (A-G, Chart 1 ), the 2-iminophenolates $\mathbf{A}$ constitute the most studied class of pro-initiator and indeed are highly effective in the presence of benzyl alcohol for the ROP of $\varepsilon-C L$ and LA [12-15]. They can also efficiently promote the copolymerization of rac-lactide and glycolide [16], rac- $\beta$-butyrolactone and $L$-lactide [17] and the random copolymerization of $r a c-L A$ and $\varepsilon$-CL with various degrees of control [18]. Related bimetallic aluminum complexes incorporating two linked iminophenolate units have also been evaluated in the ROP of cyclic esters [19]. The dialkylaluminum aminophenolate complexes B have been shown as effective (pro-)initiators for the ROP of $\varepsilon$-CL and epoxides [20-23], while their dinuclear analogues showed higher activity [22,23]; good selectivity for the ROP of rac-LA have also been noted for some B-type systems [24]. Other aluminum phenolates such as dialkylaluminum 2-imidazolylphenolates (C) and 2-benzoxazole phenolates (D) have also been reported as efficient (pro-)initiators for the ROP of rac-lactide and $\varepsilon$-CL $[25,26]$. The $\beta$-ketiminato-aluminum complex E showed good efficiency for the random copolymerization of LA and $\varepsilon$-CL $[18,27]$, while the fluorinated alkoxy-imino aluminum species $\mathbf{F}$ are active for the ROP of $\varepsilon$-CL [28]. Conversely, the amido-phosphinoxide aluminum complex $\mathrm{G}$ showed virtually no activity for the ROP of $\varepsilon$-CL [29].

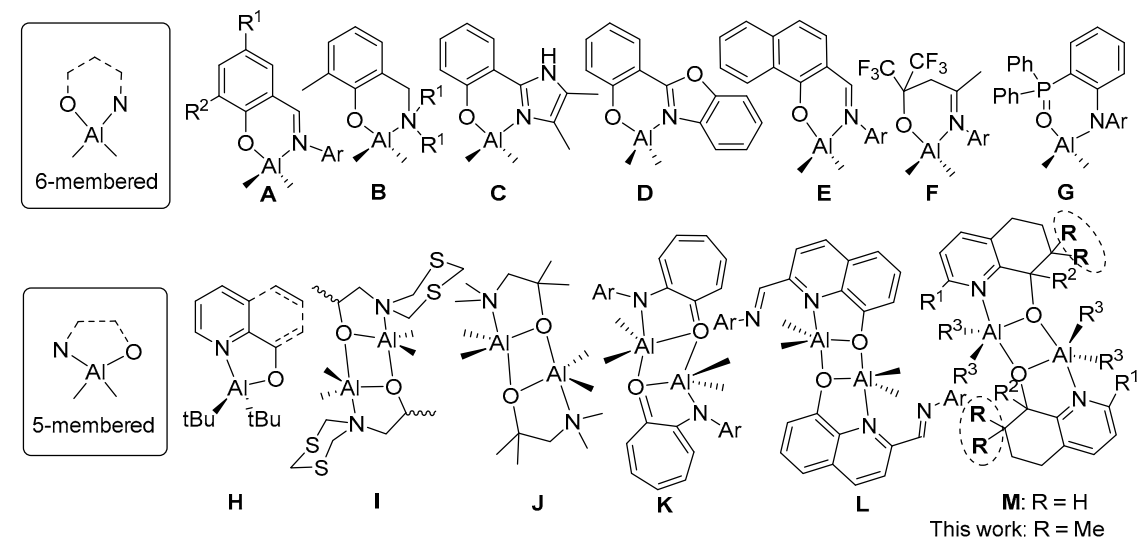

Chart 1. Reported $\mathrm{N}^{\wedge} \mathrm{O}-$ bidentate aluminum dialkyl complexes.

In comparison with the six-membered ring examples highlighted above (A-G, Chart 1$)$, those based on five-membered $\mathrm{N}^{\wedge} \mathrm{O}$-chelate rings have been considerably less studied as ROP (pro-)initiators, though a tendency to form dimeric species is a feature of their structural chemistry $(\mathbf{H}-\mathbf{M}$, Chart 1$)$. Nevertheless, a recent report has shown that five-membered ring aluminum complexes can display significantly higher polymerization rates when compared to their six-membered ring counterparts (e.g., two to three-fold increase for $\varepsilon$-CL polymerization) [30]. Elsewhere, $\mathrm{N}^{\wedge} \mathrm{O}-$ bidentate aluminum complexes of type $\mathbf{H}[31,32]$ and $\mathbf{J}$ have been reported but have not been the subject of ROP studies though $\mathbf{H}$ has been discussed in terms of the relative stability of its mono- and dimeric forms [33]. On the other hand, dimeric $\mathbf{J}$ was shown to catalyze the cycloaddition of $\mathrm{CO}_{2}$ with epoxides [34]. Moreover, the anilinotropone-based dimeric aluminum complexes $\mathbf{K}$ exhibited, in the presence of $\mathrm{BnOH}$, high activity in the ROP of rac-lactide [35]. Nonetheless, common to $\mathbf{J}$ and $\mathbf{K}$ it remains uncertain whether the dimeric structural forms are maintained during the polymerization or undergo dissociation to their monomeric forms. Interestingly, we have found the di- and monomeric complexes of dialkylaluminum 2-(arylimino)-quinolin-8-olates (L) display very different catalytic performance for the ROP of $\varepsilon$-CL. For example, the binuclear systems showed very low activity, while the mononuclear examples gave much higher efficiency and in a more controlled manner [36]. Furthermore, our group 
has recently synthesized a series of bimetallic dialkylaluminum hydroquinolin-8-olates $\left(\mathbf{M}: \mathrm{R}^{1}=\mathrm{H}\right.$, Chart 1) that showed good activity only at high temperature for the ROP of $\varepsilon$-CL. By using variable temperature ${ }^{1} \mathrm{H}$ and ${ }^{27} \mathrm{Al} \mathrm{NMR}$ spectroscopy, we proposed that the di-aluminum complexes partly dissociated into mononuclear species at high temperature [37]. However, these complexes displayed only poor solubility at room temperature which precluded full assignments of their peaks in their ${ }^{1} \mathrm{H}$ NMR spectra.

With a view to improve the solubility of $\mathbf{M}$ (Chart 1 ) and to re-investigate the nuclearity of the active initiator, we report herein the introduction of two methyl groups at the 7-position of the ligand framework. In particular, we report a series of bimetallic aluminum 5,6-dihydro-7, 7-dimethylquinolin-8-olates, [\{2- $\left.\left.\mathrm{R}^{1}-7,7-\mathrm{Me}_{2}-8-\mathrm{R}^{2} \mathrm{C}_{9} \mathrm{H}_{6} \mathrm{~N}-8-\mathrm{O}\right\} \mathrm{AlR}^{3}{ }_{2}\right]_{2}\left(\mathrm{R}^{1}=\mathrm{R}^{2}=\mathrm{H}, \mathrm{R}^{3}=\mathrm{Me}\right.$; $\mathrm{R}^{1}=\mathrm{R}^{2}=\mathrm{H}, \mathrm{R}^{3}=\mathrm{Et} ; \mathrm{R}^{1}=\mathrm{R}^{2}=\mathrm{H}, \mathrm{R}^{3}=i$-Bu; $\mathrm{R}^{1}=\mathrm{Cl}, \mathrm{R}^{2}=\mathrm{H}, \mathrm{R}^{3}=\mathrm{Me} ; \mathrm{R}^{1}=\mathrm{H}, \mathrm{R}^{2}=\mathrm{R}^{3}=\mathrm{Me}$; $\mathrm{R}^{1}=\mathrm{Cl}, \mathrm{R}^{2}=\mathrm{R}^{3}=\mathrm{Me}$ ), that differ in the substitution pattern at the $\mathrm{R}^{1}, \mathrm{R}^{2}$ and $\mathrm{R}^{3}$ positions. A full evaluation of these complexes as either initiators or pro-initiators for the ROP of $\varepsilon$-CL is conducted and these results compared with those observed using parent $\mathbf{M}$. In addition, the pathway by which pro-initiator is transformed in to the initiator is probed using both ${ }^{1} \mathrm{H}$ and ${ }^{27} \mathrm{Al} \mathrm{NMR}$ spectroscopy.

\section{Materials and Methods}

\subsection{General Considerations and Materials}

All manipulations of air or moisture-sensitive compounds were performed using standard Schlenk techniques under an atmosphere of high-purity nitrogen or using glove box techniques. Toluene was dried by refluxing it over sodium/benzophenone and distilled under nitrogen and stored over activated molecular sieves $(4 \AA)$ for $24 \mathrm{~h}$ in a glove box prior to use. $n$-Hexane and $\mathrm{CDCl}_{3}$ were dried over $\mathrm{CaH}_{2}$ for $48 \mathrm{~h}$, distilled under nitrogen and stored over activated molecular sieves $(4 \AA)$ in a glove box prior to use. Solutions of $\mathrm{Me}_{3} \mathrm{Al}$ (1.0 $\mathrm{M}$ in toluene), $\mathrm{Et}_{3} \mathrm{Al}$ (1.0 $\mathrm{M}$ in toluene), $i-\mathrm{Bu}_{3} \mathrm{Al}$ (1.0 M in toluene) and methyllithium (1.0 M in toluene) were purchased from Aldrich and used as received. Elemental analyses were performed using a PE2400II Series instrument (Perkin-Elmer Co., Shanghai, China). The ${ }^{1} \mathrm{H}$ and ${ }^{13} \mathrm{C}$ NMR spectra were recorded on a Bruker DMX-400/300 MHz (Karlsruhe, Germany) spectrometer using TMS as an internal standard; $\delta$ values are given in ppm and $J$ values in Hz. The ${ }^{27}$ Al NMR spectra were recorded on Bruker 500 MHZ (Beijing, China) spectrometer. The NMR spectra of the complexes and ligands were recorded in $\mathrm{CDCl}_{3}$ at room temperature. IR spectra were recorded on a Perkin-Elmer System 2000 (Shanghai, China) FT-IR spectrometer. The GPC measurements were performed using a set-up based on a Waters-1515 HPLC pump, a Waters 2414 (Beijng, China) refractive index detector and a combination of Styragel HT-2, HT-3 and HT-4 columns, the effective molar mass ranges of which are 100-10,000, 500-30,000 and 5000-600,000, respectively. THF was used as the eluent (flow rate: $1 \mathrm{~mL} / \mathrm{min}$, at $35^{\circ} \mathrm{C}$ ). Molecular weights and molecular weight distributions were calculated using polystyrene as a standard.

\subsection{Syntheses of $2-R^{1}-7,7-M e_{2}, 8-R^{2} \mathrm{C}_{9} H_{6} \mathrm{~N}-8-\mathrm{OH}(\mathrm{L})$}

$\mathrm{R}^{1}=\mathrm{R}^{2}=\mathrm{H}$ L1. A mixture of 5,6-dihydro-7,7-dimethylquinolin-8-one $(0.88 \mathrm{~g}, 5.0 \mathrm{mmol})$ and sodium borohydride $(0.19 \mathrm{~g}, 5.0 \mathrm{mmol})$ was dissolved in methanol $(30 \mathrm{~mL})$ in a $100 \mathrm{~mL}$ round flask and stirred for $5 \mathrm{~h}$ at room temperature. The reaction was then quenched with water, extracted with dichloromethane and the organic phase dried over anhydrous magnesium sulfate and filtered. The filtrate was collected and the solvent evaporated under reduced pressure to give $\mathbf{L 1}$ as a light yellow solid. Yield: $0.86 \mathrm{~g}(96 \%) .{ }^{1} \mathrm{H}$ NMR $\left(400 \mathrm{MHz}, \mathrm{CDCl}_{3}, \mathrm{ppm}\right): \delta 8.40(\mathrm{~d}, J=6.0 \mathrm{~Hz}, 1 \mathrm{H}, \mathrm{Py}-\mathrm{H})$, $7.41(\mathrm{~d}, J=8.0 \mathrm{~Hz}, 1 \mathrm{H}, \mathrm{Py}-H), 7.14-7.11(\mathrm{~m}, 1 \mathrm{H}, \mathrm{Py}-\mathrm{H}), 4.38(\mathrm{~s}, 1 \mathrm{H}, \mathrm{CH}-\mathrm{OH}), 4.31(\mathrm{~s}, 1 \mathrm{H}, \mathrm{OH}), 2.92-2.79$ $\left(\mathrm{m}, 2 \mathrm{H}, \mathrm{CH}_{2}\right), 1.75-1.65\left(\mathrm{~m}, 2 \mathrm{H}, \mathrm{CH}_{2}\right), 1.19\left(\mathrm{~s}, 3 \mathrm{H}, \mathrm{CH}_{3}\right), 0.85\left(\mathrm{~s}, 3 \mathrm{H}, \mathrm{CH}_{3}\right) .{ }^{13} \mathrm{C} \mathrm{NMR}(100 \mathrm{MHz}$, $\left.\mathrm{CDCl}_{3}, \mathrm{ppm}\right): \delta 157.3,146.3,136.5,130.4,122.0,76.2,34.0,33.3,27.5,24.8,18.9$. IR $\left(\mathrm{cm}^{-1}\right): 3117(\mathrm{~m})$, $2953(\mathrm{~m}), 2922(\mathrm{~m}), 2859(\mathrm{w}), 2714(\mathrm{w}), 1583(\mathrm{~m}), 1446(\mathrm{~m}), 1377(\mathrm{~m}), 1359(\mathrm{~m}), 1307(\mathrm{w}), 1260(\mathrm{~m})$, 
$1160(\mathrm{~m}), 1107(\mathrm{~m}), 1042(\mathrm{~s}), 981(\mathrm{~m}), 936(\mathrm{w}), 904(\mathrm{w}), 857(\mathrm{w}), 788(\mathrm{~s}), 751(\mathrm{~m}), 713(\mathrm{~m})$. Anal. Calcd for $\mathrm{C}_{11} \mathrm{H}_{15} \mathrm{NO}: \mathrm{C}, 74.54 ; \mathrm{H}, 8.53 ; \mathrm{N}, 7.90 \%$. Found: $\mathrm{C}, 74.40 ; \mathrm{H}, 8.53 ; \mathrm{N}, 7.81 \%$.

$\mathrm{R}^{1}=\mathrm{Cl}, \mathrm{R}^{2}=\mathrm{H}$ L2. Using a similar procedure to that described for L1, but with 2-chloro-5,6-dihydro-7,7-dimethylquinolin-8-one as the ketone, gave $\mathbf{L} 2$ as a white powder. Yield: $1.03 \mathrm{~g}(97 \%) .{ }^{1} \mathrm{H}$ NMR $\left(400 \mathrm{MHz}, \mathrm{CDCl}_{3}, \mathrm{ppm}\right): \delta 7.38(\mathrm{~d}, J=8.0 \mathrm{~Hz}, 1 \mathrm{H}, \mathrm{Py}-H), 7.14(\mathrm{~d}, J=8.0 \mathrm{~Hz}, 1 \mathrm{H}$, Py-H), 4.27 (s, 1H, CH-OH), $3.76(\mathrm{~s}, 1 \mathrm{H}, \mathrm{OH}), 2.85-2.69\left(\mathrm{~m}, 2 \mathrm{H}, \mathrm{CH}_{2}\right), 1.71-1.67\left(\mathrm{~m}, 2 \mathrm{H}, \mathrm{CH}_{2}\right), 1.16$ $\left(\mathrm{s}, 3 \mathrm{H}, \mathrm{CH}_{3}\right), 0.87\left(\mathrm{~s}, 3 \mathrm{H}, \mathrm{CH}_{3}\right) .{ }^{13} \mathrm{C} \mathrm{NMR}\left(100 \mathrm{MHz}, \mathrm{CDCl}_{3}, \mathrm{ppm}\right): \delta 158.3,148.6,139.6,129.0,123.0$, 76.0, 34.0, 33.3, 27.5, 24.3, 19.0. IR ( $\left.\mathrm{cm}^{-1}\right)$ : $3281(\mathrm{~m}), 2943(\mathrm{~m}), 2905(\mathrm{~m}), 2865(\mathrm{w}), 1570(\mathrm{~m}), 1469(\mathrm{~m})$, $1440(\mathrm{~m}), 1416(\mathrm{w}), 1377(\mathrm{~m}), 1363(\mathrm{~m}), 1256(\mathrm{~m}), 1230(\mathrm{w}), 1197(\mathrm{w}), 1164(\mathrm{~m}), 1131(\mathrm{w}), 1090(\mathrm{~m})$, $1033(\mathrm{~s}), 946(\mathrm{~m}), 904(\mathrm{w}), 870(\mathrm{w}), 842(\mathrm{~m}), 789(\mathrm{~s}), 756(\mathrm{~m}), 727(\mathrm{~m})$. Anal. Calcd for $\mathrm{C}_{11} \mathrm{H}_{14} \mathrm{ClNO}$ : C, $62.41 ; \mathrm{H}, 6.67 ; \mathrm{N}, 6.62 \%$. Found: C, 62.58; H, 6.59; N, 6.43\%.

$\mathrm{R}^{1}=\mathrm{H}, \mathrm{R}^{2}=$ Me L3. Methyllithium $(5.0 \mathrm{~mL}, 5.0 \mathrm{mmol}, 1.0 \mathrm{M}$ solution in toluene) was added dropwise to a stirred solution of 5,6-dihydro-7,7-dimethylquinolin-8-one $(0.88 \mathrm{~g}, 5.0 \mathrm{mmol})$ in toluene $(10.0 \mathrm{~mL})$ at $-30{ }^{\circ} \mathrm{C}$. The reaction mixture was allowed to warm slowly to room temperature and stirred overnight. Following quenching with water and extraction with dichloromethane, the organic phase was dried over anhydrous magnesium sulfate and filtered. The filtrate was collected and the solvent removed under reduced pressure to give $\mathbf{L} 3$ as a white solid. Yield: $0.93 \mathrm{~g}(97 \%) .{ }^{1} \mathrm{H}$ NMR (400 MHz, CDCl 3 , ppm): $\delta 8.39$ (d, J = 4.0 Hz, 1H, Py-H), 7.38 (d, J = 8.0 Hz, 1H, Py-H), 7.11-7.08 $(\mathrm{m}, 1 \mathrm{H}, \mathrm{Py}-\mathrm{H}), 4.25(\mathrm{~s}, 1 \mathrm{H}, \mathrm{OH}), 2.90-2.74\left(\mathrm{~m}, 2 \mathrm{H}, \mathrm{CH}_{2}\right), 1.94-1.86\left(\mathrm{~m}, 1 \mathrm{H}, \mathrm{CH}_{2}\right), 1.63-1.58(\mathrm{~m}, 1 \mathrm{H}$, $\left.\mathrm{CH}_{2}\right), 1.37\left(\mathrm{~s}, 1 \mathrm{H}, \mathrm{CH}_{3}\right), 1.15\left(\mathrm{~s}, 1 \mathrm{H}, \mathrm{CH}_{3}\right), 0.93\left(\mathrm{~s}, 1 \mathrm{H}, \mathrm{CH}_{3}\right) .{ }^{13} \mathrm{C} \mathrm{NMR}\left(100 \mathrm{MHz}, \mathrm{CDCl}_{3}, \mathrm{ppm}\right): \delta 162.3$, 148.3 139.6, 128.2, 122.4, 74.7, 36.1, 33.0, 26.7, 24.5, 23.9, 21.9. IR ( $\left.\mathrm{cm}^{-1}\right): 3413(\mathrm{~m}), 2959(\mathrm{~m}), 2872(\mathrm{w})$, $1702(\mathrm{w}), 1580(\mathrm{~m}), 1447(\mathrm{~m}), 1424(\mathrm{~m}), 1386(\mathrm{~m}), 1359(\mathrm{~m}), 1336(\mathrm{w}), 1283(\mathrm{~m}), 1260(\mathrm{~m}), 1169(\mathrm{~m})$, $1131(\mathrm{~m}), 1077(\mathrm{~s}), 1018(\mathrm{~m}), 978(\mathrm{~m}), 934(\mathrm{w}), 907(\mathrm{w}), 789(\mathrm{~s})$. Anal. Calcd for $\mathrm{C}_{12} \mathrm{H}_{17} \mathrm{NO}$ : C, 75.35; $\mathrm{H}, 8.96 ; \mathrm{N}, 7.32 \%$. Found: $\mathrm{C}, 75.26 ; \mathrm{H}, 8.82 ; \mathrm{N}, 7.46 \%$.

$\mathrm{R}^{1}=\mathrm{Cl}, \mathrm{R}^{2}=$ Me L4. Using a similar procedure to that described for L3, but with 2-chloro-5, 6-dihydro-7,7-dimethylquinolin-8-one as the ketone, gave $\mathbf{L} 4$ as a white powder. Yield: $1.09 \mathrm{~g}(97 \%)$. ${ }^{1} \mathrm{H}$ NMR (400 MHz, CDCl 3 , ppm): $7.34(\mathrm{~d}, J=8.0 \mathrm{~Hz}, 1 \mathrm{H}, \mathrm{Py}-H), 7.11(\mathrm{~d}, J=8.0 \mathrm{~Hz}, 1 \mathrm{H}, \mathrm{Py}-H), 3.65$ (s, $1 \mathrm{H}, \mathrm{OH}), 2.87-2.70\left(\mathrm{~m}, 2 \mathrm{H}, \mathrm{CH}_{2}\right), 1.89-1.81\left(\mathrm{~m}, 1 \mathrm{H}, \mathrm{CH}_{2}\right), 1.65-1.59\left(\mathrm{~m}, 1 \mathrm{H}, \mathrm{CH}_{2}\right), 1.38\left(\mathrm{~s}, 3 \mathrm{H}, \mathrm{CH}_{3}\right)$, $1.12\left(\mathrm{~s}, 3 \mathrm{H}, \mathrm{CH}_{3}\right), 0.94\left(\mathrm{~s}, 3 \mathrm{H}, \mathrm{CH}_{3}\right) .{ }^{13} \mathrm{C} \mathrm{NMR}\left(100 \mathrm{MHz}, \mathrm{CDCl}_{3}, \mathrm{ppm}\right): \delta 161.4,146.5,136.5,129.2$, 121.8, 74.9, 36.2, 33.0, 27.1, 24.5, 24.4, 22.0. IR (cm $\left.{ }^{-1}\right)$ : $3477(\mathrm{~m}), 2963(\mathrm{~m}), 2924(\mathrm{~m}), 1697(\mathrm{~m}), 1572(\mathrm{~m})$, $1444(\mathrm{~m}), 1427(\mathrm{~m}), 1385(\mathrm{~m}), 1360(\mathrm{~m}), 1328(\mathrm{~m}), 1316(\mathrm{w}), 1261(\mathrm{~m}), 1191(\mathrm{w}), 1166(\mathrm{~m}), 1127(\mathrm{~m})$, 1069 (s), $1016(\mathrm{~m}), 932(\mathrm{w}), 854(\mathrm{w}), 815$ (s), $748(\mathrm{~m})$. Anal. Calcd for $\mathrm{C}_{12} \mathrm{H}_{16} \mathrm{ClNO}$ : C, 63.86; H, 7.15; N, $6.21 \%$. Found: $\mathrm{C}, 63.45 ; \mathrm{H}, 7.28 ; \mathrm{N}, 6.46 \%$.

\subsection{Syntheses of $\left[\left\{2-R^{1}-7,7-\mathrm{Me}_{2}-8-R^{2} \mathrm{C}_{9} \mathrm{H}_{6} \mathrm{~N}-8-\mathrm{O}\right\} \mathrm{AlR}_{3}{ }^{2}\right]_{2}(\mathrm{C})$}

$\mathrm{R}^{1}=\mathrm{R}^{2}=\mathrm{H}, \mathrm{R}^{3}=\mathrm{Me} \mathbf{C}$. $\mathrm{Me}_{3} \mathrm{Al}(5.0 \mathrm{~mL}, 5.0 \mathrm{mmol}, 1.0 \mathrm{M}$ solution in toluene) was added dropwise to a stirred solution of $\mathbf{L} 1(0.89 \mathrm{~g}, 5.0 \mathrm{mmol})$ in toluene $(10 \mathrm{~mL})$ at $-78{ }^{\circ} \mathrm{C}$. The resulting solution was allowed to warm slowly to room temperature and stirred for $3 \mathrm{~h}$. Following concentration of the reaction mixture to ca. $1 \mathrm{~mL}$, hexane $(10 \mathrm{~mL})$ was added to induce precipitation. The precipitate was filtered affording $\mathbf{C} 1$ as a white powder. Yield: $0.75 \mathrm{~g}, 65 \% .{ }^{1} \mathrm{H}$ NMR $\left(400 \mathrm{MHz}\right.$, toluene- $\left.d_{8}, \mathrm{ppm}\right)$ : $\delta 7.44(\mathrm{~d}, J=8.0 \mathrm{~Hz}, 1 \mathrm{H}, \mathrm{Py}-H), 6.63(\mathrm{~d}, J=8.0 \mathrm{~Hz}, 1 \mathrm{H}, \mathrm{Py}-H), 6.47-6.41(\mathrm{~m}, 1 \mathrm{H}, \mathrm{Py}-H), 4.58(\mathrm{~s}, 1 \mathrm{H}$, $\mathrm{CH}-\mathrm{O}), 2.07-1.98\left(\mathrm{~m}, 2 \mathrm{H}, \mathrm{CH}_{2}\right), 1.52\left(\mathrm{~s}, 3 \mathrm{H}, \mathrm{CH}_{3}\right), 1.21-1.13\left(\mathrm{~m}, 2 \mathrm{H}, \mathrm{CH}_{2}\right), 0.68\left(\mathrm{~s}, 3 \mathrm{H}, \mathrm{CH}_{3}\right),-0.12$ $\left(\mathrm{s}, 3 \mathrm{H}, \mathrm{Al}-\mathrm{CH}_{3}\right),-0.26\left(\mathrm{~s}, 3 \mathrm{H}, \mathrm{Al}-\mathrm{CH}_{3}\right) .{ }^{13} \mathrm{C} \mathrm{NMR}\left(100 \mathrm{MHz}\right.$, toluene- $\left.d_{8}, \mathrm{ppm}\right): \delta 157.0,141.7,140.1$, 134.2, 124.0, 82.1, 36.2, 35.8, 30.1, 24.2, 17.7, -3.26. Anal. Calcd for $\mathrm{C}_{13} \mathrm{H}_{20} \mathrm{AlNO}$ :, $66.93 ; \mathrm{H}, 8.64 ; \mathrm{N}$, $6.00 \%$. Found: C, $67.13 ; \mathrm{H}, 8.77 ; \mathrm{N}, 5.88 \%$.

$\mathrm{R}^{1}=\mathrm{R}^{2}=\mathrm{H}, \mathrm{R}^{3}=\mathrm{Et} \mathbf{C}$. Using a similar procedure to that described for $\mathbf{C} 1$, but with $\mathrm{Et}_{3} \mathrm{Al}(1.0 \mathrm{M}$ solution in toluene) as the alkyl aluminum reagent, gave $\mathbf{C} 2$ as a white powder. Yield: $0.55 \mathrm{~g}, 42 \%$. ${ }^{1} \mathrm{H}$ NMR $\left(400 \mathrm{MHz}\right.$, toluene- $\left.d_{8}, \mathrm{ppm}\right): \delta 8.11(\mathrm{~d}, J=4.0 \mathrm{~Hz}, 1 \mathrm{H}, \mathrm{Py}-H), 6.74(\mathrm{~d}, J=5.6 \mathrm{~Hz}, 1 \mathrm{H}, \mathrm{Py}-H)$, $6.57(\mathrm{t}, J=6.0 \mathrm{~Hz}, 1 \mathrm{H}, \mathrm{Py}-\mathrm{H}), 5.01(\mathrm{~s}, 1 \mathrm{H}, \mathrm{CH}-\mathrm{O}), 2.34-2.22\left(\mathrm{~m}, 2 \mathrm{H}, \mathrm{CH}_{2}\right), 1.54\left(\mathrm{~s}, 3 \mathrm{H}, \mathrm{CH}_{3}\right), 1.45-1.37$ $\left(\mathrm{m}, 1 \mathrm{H}, \mathrm{Al}-\mathrm{CH}_{2} \mathrm{CH}_{3}\right), 1.30-1.25\left(\mathrm{~m}, 2 \mathrm{H}, \mathrm{CH}_{2}\right), 1.21-1.15\left(\mathrm{~m}, 3 \mathrm{H}, \mathrm{Al}-\mathrm{CH}_{2} \mathrm{CH}_{3}\right), 0.94\left(\mathrm{~s}, 3 \mathrm{H}, \mathrm{CH}_{3}\right.$ and 
$\left.\mathrm{Al}-\mathrm{CH}_{2} \mathrm{CH}_{3}\right), 0.51-0.44\left(\mathrm{~m}, 2 \mathrm{H}, \mathrm{Al}-\mathrm{CH}_{2} \mathrm{CH}_{3}\right), 0.35-0.26\left(\mathrm{~m}, 2 \mathrm{H}, \mathrm{Al}-\mathrm{CH}_{2} \mathrm{CH}_{3}\right) .{ }^{13} \mathrm{C} \mathrm{NMR}(100 \mathrm{MHz}$, toluene- $d_{8}$, ppm): $\delta 164.4,158.5,142.5,132.1,122.7,80.9,36.7,36.5,30.2,24.9,18.6,11.4,11.3,1.9$. Anal. Calcd for $\mathrm{C}_{15} \mathrm{H}_{24}$ AlNO: C, 68.94; H, 9.26; N, 5.36\%. Found: C, 69.17; H, 9.44; N, 5.23\%.

$\mathrm{R}^{1}=\mathrm{R}^{2}=\mathrm{H}, \mathrm{R}^{3}=i$-Bu C3. Using a similar procedure to that described for $\mathbf{C} \mathbf{1}$, but with $i$ - $\mathrm{Bu}_{3} \mathrm{Al}$ (1.0 M solution in toluene) as the alkyl aluminum reagent, gave $\mathbf{C} 3$ as a white powder. Yield: $0.33 \mathrm{~g}$, 21\%. ${ }^{1} \mathrm{H}$ NMR $\left(400 \mathrm{MHz}, \mathrm{CDCl}_{3}, \mathrm{ppm}\right): \delta 8.23(\mathrm{~d}, J=8.0 \mathrm{~Hz}, 1 \mathrm{H}, \mathrm{Py}-\mathrm{H}), 7.67(\mathrm{~d}, J=4.0 \mathrm{~Hz}, 1 \mathrm{H}$, Py-H), 7.38-7.34 (m, 1H, Py-H), 4.61 (s, $1 \mathrm{H}, \mathrm{CH}-\mathrm{O}), 2.94-2.80\left(\mathrm{~m}, 2 \mathrm{H}, \mathrm{CH}_{2}\right), 1.86-1.67\left(\mathrm{~m}, 3 \mathrm{H}, \mathrm{CH}_{2}\right.$ and $\left.\mathrm{Al}-\mathrm{CH}_{2} \mathrm{CH}\left(\mathrm{CH}_{3}\right)_{2}\right), 1.23\left(\mathrm{~s}, 3 \mathrm{H}, \mathrm{CH}_{3}\right), 0.90-0.75\left(\mathrm{~m}, 12 \mathrm{H}, \mathrm{Al}-\mathrm{CH}_{2} \mathrm{CH}\left(\mathrm{CH}_{3}\right)_{2}\right), 0.73\left(\mathrm{~s}, 3 \mathrm{H}, \mathrm{CH}_{3}\right)$, $-0.07-0.09\left(\mathrm{~m}, 4 \mathrm{H}, \mathrm{Al}-\mathrm{CH}_{2} \mathrm{CH}\left(\mathrm{CH}_{3}\right)_{2}\right) .{ }^{13} \mathrm{C} \mathrm{NMR}\left(100 \mathrm{MHz}, \mathrm{CDCl}_{3}\right): \delta$ 164.0, 142.2, 139.6, 133.6, 122.8, 80.4, 35.3, 34.3, 28.2, 28.1, 28.0, 27.9, 26.2, 26.0, 24.5, 22.6, 17.4. Anal. Calcd for $\mathrm{C}_{19} \mathrm{H}_{32} \mathrm{AlNO}$ : C, $71.89 ; \mathrm{H}, 10.16 ; \mathrm{N}, 4.41 \%$. Found: C, 71.56; H, 10.52; N, 4.67\%.

$\mathrm{R}^{1}=\mathrm{Cl}, \mathrm{R}^{2}=\mathrm{H}, \mathrm{R}^{3}=\mathrm{Me} \mathbf{C} 4$. Using a similar procedure to that described for $\mathbf{C} \mathbf{1}$, but with $\mathbf{L} \mathbf{2}$ as the pro-ligand, gave $\mathrm{C} 4$ as a white powder. Yield: $1.14 \mathrm{~g}, 85 \% .{ }^{1} \mathrm{H} \mathrm{NMR}\left(400 \mathrm{MHz}, \mathrm{CDCl}_{3}, \mathrm{ppm}\right): 7.74(\mathrm{~d}$, $J=8.0 \mathrm{~Hz}, 1 \mathrm{H}, \mathrm{Py}-\mathrm{H}), 7.44(\mathrm{~d}, J=8.0 \mathrm{~Hz}, 1 \mathrm{H}, \mathrm{Py}-\mathrm{H}), 4.92(\mathrm{~s}, 1 \mathrm{H}, \mathrm{CH}-\mathrm{O}), 2.85-2.81\left(\mathrm{~m}, 2 \mathrm{H}, \mathrm{CH}_{2}\right), 1.92-1.84$ $\left(\mathrm{m}, 1 \mathrm{H}, \mathrm{CH}_{2}\right), 1.71-1.65\left(\mathrm{~m}, 1 \mathrm{H}, \mathrm{CH}_{2}\right), 1.51\left(\mathrm{~s}, 3 \mathrm{H}, \mathrm{CH}_{3}\right), 0.84\left(\mathrm{~s}, 3 \mathrm{H}, \mathrm{CH}_{3}\right),-0.58\left(\mathrm{~s}, 6 \mathrm{H}, \mathrm{Al}-\mathrm{CH}_{3}\right) .{ }^{13} \mathrm{C} \mathrm{NMR}$ (100 MHz, $\mathrm{CDCl}_{3}$, ppm): $\delta 158.4,145.5,142.4,132.8,126.7,81.5,36.1,35.8,31.6,29.5,23.9,18.1,14.3,-4.5$. Anal. Calcd for $\mathrm{C}_{13} \mathrm{H}_{19} \mathrm{AlClNO}$ : C, 58.32; H, 7.15; N, 5.23\%. Found: $\mathrm{C}, 58.11 ; \mathrm{H}, 7.24 ; \mathrm{N}, 5.38 \%$.

$\mathrm{R}^{1}=\mathrm{H}, \mathrm{R}^{2}=\mathrm{R}^{3}=$ Me $\mathbf{C}$. Using a similar procedure to that described for $\mathbf{C} \mathbf{1}$, but with $\mathbf{L} 3$ as the pro-ligand, gave $\mathrm{C} 5$ as a white powder. Yield: $0.36 \mathrm{~g}, 29 \% .{ }^{1} \mathrm{H}$ NMR $\left(400 \mathrm{MHz}\right.$, Toluene- $\left.d_{8}, \mathrm{ppm}\right)$ : $\delta 7.49(\mathrm{~d}, J=4.0 \mathrm{~Hz}, 1 \mathrm{H}, \mathrm{Py}-H), 6.74(\mathrm{~d}, J=8.0 \mathrm{~Hz}, 1 \mathrm{H}, \mathrm{Py}-H), 6.51-6.48(\mathrm{~m}, 1 \mathrm{H}, \mathrm{Py}-H), 2.21-2.18(\mathrm{~m}$, $\left.2 \mathrm{H}, \mathrm{CH}_{2}\right), 1.56-1.51\left(\mathrm{~m}, 1 \mathrm{H}, \mathrm{CH}_{2}\right), 1.49\left(\mathrm{~s}, 3 \mathrm{H}, \mathrm{CH}_{3}\right), 1.43\left(\mathrm{~s}, 3 \mathrm{H}, \mathrm{CH}_{3}\right), 1.22-1.15\left(\mathrm{~m}, 1 \mathrm{H}, \mathrm{CH}_{2}\right), 0.83(\mathrm{~s}$, $\left.3 \mathrm{H}, \mathrm{CH}_{3}\right),-0.10\left(\mathrm{~s}, 3 \mathrm{H}, \mathrm{Al}-\left(\mathrm{CH}_{3}\right)_{2}\right),-0.30\left(\mathrm{~s}, 3 \mathrm{H}, \mathrm{Al}-\left(\mathrm{CH}_{3}\right)_{2}\right) .{ }^{13} \mathrm{C}$ NMR $\left(100 \mathrm{MHz}\right.$, toluene- $\left.d_{8}, \mathrm{ppm}\right)$ : 反 163.2, 143.4, 142.5, 134.3, 126.0, 87.7, 40.3, 36.72, 28.9, 27.8, 25.2, 23.3, -2.09, -2.92. Anal. Calcd. for $\mathrm{C}_{14} \mathrm{H}_{22}$ AlNO: C, $67.99 ; \mathrm{H}, 8.97 ; \mathrm{N}, 5.66 \%$. Found: $\mathrm{C}, 68.20 ; \mathrm{H}, 8.83 ; \mathrm{N}, 5.79 \%$.

$\mathrm{R}^{1}=\mathrm{Cl}, \mathrm{R}^{2}=\mathrm{R}^{3}=\mathrm{Me} \mathbf{C 6}$. Using a similar procedure to that described for $\mathbf{C} \mathbf{1}$, but with $\mathbf{L} 4$ as the pro-ligand, gave $\mathrm{C} 6$ as a white powder. Yield: $0.48 \mathrm{~g}, 34 \% .{ }^{1} \mathrm{H} \mathrm{NMR}\left(300 \mathrm{MHz}, \mathrm{CDCl}_{3}, \mathrm{ppm}\right): \delta 7.52$ $(\mathrm{d}, J=9.0 \mathrm{~Hz}, 1 \mathrm{H}, \mathrm{Py}-H), 7.28(\mathrm{t}, J=9.0 \mathrm{~Hz}, 1 \mathrm{H}, \mathrm{Py}-H), 2.88-2.83\left(\mathrm{~m}, 2 \mathrm{H}, \mathrm{CH}_{2}\right), 2.02-1.91\left(\mathrm{~m}, 1 \mathrm{H}, \mathrm{CH}_{2}\right)$, 1.70-1.62 (m, 1H, CH $\left.\mathrm{CH}_{2}\right), 1.44\left(\mathrm{~s}, 3 \mathrm{H}, \mathrm{CH}_{3}\right), 1.29\left(\mathrm{~s}, 3 \mathrm{H}, \mathrm{CH}_{3}\right), 0.91\left(\mathrm{~s}, 3 \mathrm{H}, \mathrm{CH}_{3}\right),-0.69\left(\mathrm{~s}, 6 \mathrm{H}, \mathrm{Al}-\left(\mathrm{CH}_{3}\right)_{2}\right)$. ${ }^{13} \mathrm{C}$ NMR (100 MHz, $\mathrm{CDCl}_{3}$, ppm): $\delta 161.1,146.7,141.1,128.2,125.0,81.0,37.4,33.8,27.3,25.1,23.2,21.0$, 14.4, -8.3. Anal. Calcd for $\mathrm{C}_{14} \mathrm{H}_{21} \mathrm{AlClNO}$ C, 59.68; H, 7.51; N, 4.97\%. Found: $\mathrm{C}, 59.55 ; \mathrm{H}, 7.32 ; \mathrm{N}, 4.85 \%$.

\subsection{X-ray Crystallographic Studies}

Single crystal X-ray diffraction data for $\mathbf{C} \mathbf{1}$ and $\mathbf{C 4}$ were collected on a Rigaku Sealed Tube CCD (Saturn 724+) diffractometer (Tokyo, Japan) with graphite monochromated Mo-K $\alpha$ radiation $(\lambda=0.71073 \AA)$ at $293(2) \mathrm{K}$. Cell parameters were obtained by global refinement of the positions of all collected reflections. Intensities were corrected for Lorentz and polarization effects and empirical absorption. The structures were solved by direct methods and refined by full-matrix least squares on $F^{2}$. All hydrogen atoms were placed in calculated positions. Structure solution and refinement were performed using the SHELXTL-97 package $[38,39]$. Details of the X-ray structure determinations and refinements are provided in Table 1. The Cambridge Crystallographic Data Centre CCDC 1851289 and 1851290 for $\mathbf{C} \mathbf{1}$ and $\mathbf{C 4}$, respectively, contain the supplementary crystallographic data for the paper. These data can be obtained free of charge from the Cambridge Crystallographic Data Centre (Cambridge, UK).

Table 1. Crystal data and structure refinement for $\mathrm{C} 1$ and $\mathrm{C4}$.

\begin{tabular}{ccc}
\hline & $\mathrm{C} \mathbf{1}$ & $\mathrm{C} 4$ \\
\hline Empirical formula & $\mathrm{C}_{26} \mathrm{H}_{40} \mathrm{Al}_{2} \mathrm{~N}_{2} \mathrm{O}_{2}$ & $\mathrm{C}_{13} \mathrm{H}_{19} \mathrm{AlNOCl}$ \\
Formula weight & 466.56 & 267.72 \\
Crystal color & colorless & colorless \\
Temperature $(\mathrm{K})$ & $293(2)$ & $293(2)$ \\
Wavelength $(\AA)$ & 0.71073 & 0.71073 \\
Crystal system & monoclinic & monoclinic \\
\hline
\end{tabular}


Table 1. Cont.

\begin{tabular}{|c|c|c|}
\hline & $\mathrm{C} 1$ & $\mathrm{C} 4$ \\
\hline space group & $\mathrm{P} 2 / \mathrm{n}$ & $\mathrm{P} 21 / \mathrm{n}$ \\
\hline a $(\AA)$ & 9.762(2) & $10.934(5)$ \\
\hline $\mathrm{b}(\AA)$ & $9.773(2)$ & $9.902(4)$ \\
\hline$c(\AA)$ & $13.676(3)$ & $13.019(6)$ \\
\hline$\alpha\left(^{\circ}\right)$ & 90.00 & 90.00 \\
\hline$\beta\left({ }^{\circ}\right)$ & 90.39(3) & 100.391(5) \\
\hline$\gamma\left({ }^{\circ}\right)$ & 90.00 & 90.00 \\
\hline Volume $\left(\AA^{3}\right)$ & $1304.7(5)$ & $1386.5(11)$ \\
\hline Z & 2 & 4 \\
\hline $\mathrm{D}_{\text {calc }}\left(\mathrm{Mg} \mathrm{m}^{-3}\right)$ & 1.188 & 1.283 \\
\hline$\mu\left(\mathrm{mm}^{-1}\right)$ & 0.136 & 0.323 \\
\hline $\mathrm{F}(000)$ & 504.0 & 568.0 \\
\hline Crystal size $(\mathrm{mm})$ & $0.49 \times 0.29 \times 0.21$ & $0.64 \times 0.38 \times 0.16$ \\
\hline$\theta$ range $\left(^{\circ}\right)$ & $\begin{array}{l}5.14-54.96 \\
-12<\mathrm{h}<11\end{array}$ & $\begin{array}{c}4.49-54.94 \\
-14<\mathrm{h}<14\end{array}$ \\
\hline Limiting indices & $\begin{array}{l}-12 \leq \mathrm{k} \leq 12 \\
-17 \leq 1 \leq 17\end{array}$ & $\begin{array}{l}-12 \leq \mathrm{k} \leq 12 \\
-16 \leq 1 \leq 16\end{array}$ \\
\hline No.of rflns collected & 13159 & $1 \overline{4} 47 \overline{3}$ \\
\hline No. of unique rflns $R_{\text {int }}$ & $2963(0.0301)$ & $3144(0.0526)$ \\
\hline Completeness to $\theta(\%)$ & $99.0 \%$ & $99.3 \%$ \\
\hline Goodness-of-fit on $\mathrm{F}^{2}$ & 1.198 & 1.137 \\
\hline Final $\mathrm{R}$ indices $[\mathrm{I}>2 \sigma(\mathrm{I})]$ & $\begin{array}{c}\mathrm{R}_{1}=0.0372 \\
\mathrm{wR}_{2}=0.1078\end{array}$ & $\mathrm{R}_{1}=0.0652, \mathrm{wR}_{2}=0.1592$ \\
\hline $\mathrm{R}$ indices (all data) & $\begin{array}{r}\mathrm{R}_{1}=0.0429 \\
\mathrm{wR}_{2}=0.1246\end{array}$ & $\mathrm{R}_{1}=0.0678, \mathrm{wR}_{2}=0.1621$ \\
\hline Largest diff. peak and hole $\left(\mathrm{e} \AA^{-3}\right)$ & $0.44 /-0.48$ & $0.36 /-0.34$ \\
\hline
\end{tabular}

\subsection{General Procedure for $\varepsilon$-Caprolactone Polymerization}

A typical polymerization procedure in the presence of one equivalent of benzyl alcohol is outlined as follows. Precatalyst C1 $(0.0047 \mathrm{~g}, 0.020 \mathrm{mmol})$ was dissolved in toluene $(2 \mathrm{~mL})$ in a Schlenk flask at room temperature and a solution of benzyl alcohol $(0.020 \mathrm{mmol})$ in toluene added and the mixture stirred at room temperature for $5 \mathrm{~min}$. The flask was then placed in a temperature-controlled oil bath preheated to $90^{\circ} \mathrm{C}$ and $\varepsilon$-CL $(0.571 \mathrm{~g}, 5.0 \mathrm{mmol})$ was injected. After the solution was stirred for the pre-determined time, the polymerization was terminated by the addition of glacial acetic acid (ca. $0.2 \mathrm{~mL}$ ). The resulting viscous solution was diluted with dichloromethane and then transferred to a beaker containing cold methanol $(100 \mathrm{~mL})$ with stirring. The resultant polymer was collected on filter paper and dried under reduced pressure to give a white solid.

\section{Results and Discussion}

\subsection{Synthesis and Characterization of $\mathbf{C 1}-\mathbf{C 6}$}

Based on previous synthetic approaches, reduction of the 5,6-dihydro-7,7-dimethylquinolin-8 -ones, 2- $\mathrm{R}^{1}-7,7-\mathrm{Me}_{2} \mathrm{C}_{9} \mathrm{H}_{6} \mathrm{~N}-8-\mathrm{O}\left(\mathrm{R}^{1}=\mathrm{H} ; \mathrm{R}^{1}=\mathrm{Cl}\right)$, with $\mathrm{NaBH}_{4}$ or LiMe gave the dihydroquinolin-8-ols, 2-R ${ }^{1}-7,7-\mathrm{Me}_{2}, 8-\mathrm{R}_{2} \mathrm{C}_{9} \mathrm{H}_{6} \mathrm{~N}-8-\mathrm{OH}\left(\mathrm{R}^{1}=\mathrm{R}^{2}=\mathrm{H} \mathrm{L1} ; \mathrm{R}^{1}=\mathrm{Cl}, \mathrm{R}^{2}=\mathrm{H}\right.$ L2; $\mathrm{R}^{1}=\mathrm{H}, \mathrm{R}^{2}=\mathrm{Me} \mathrm{L3} ; \mathrm{R}^{1}=\mathrm{Cl}$, $\mathrm{R}^{2}=$ Me L4), in high yield (Scheme 1). All four compounds have been fully characterized by ${ }^{1} \mathrm{H} /{ }^{13} \mathrm{C}$ NMR, FT-IR spectroscopy as well as by elemental analysis. Treatment of $\mathbf{L 1}$ with one equivalent of $\left(\mathrm{R}^{3}\right)_{3} \mathrm{Al}\left(\mathrm{R}^{3}=\mathrm{Me}, \mathrm{Et}, i-\mathrm{Bu}\right)$ in toluene at $-78{ }^{\circ} \mathrm{C}$ afforded $\left[\left\{2-\mathrm{R}^{1}-7,7-\mathrm{Me}_{2}-8-\mathrm{R}_{2} \mathrm{C}_{9} \mathrm{H}_{6} \mathrm{~N}-8-\mathrm{O}\right\} \mathrm{AlR}^{3}{ }_{2}\right]_{2}$ $\left(\mathrm{R}^{1}=\mathrm{R}^{2}=\mathrm{H}, \mathrm{R}^{3}=\right.$ Me C1; $\left.\mathrm{R}^{1}=\mathrm{R}^{2}=\mathrm{H}, \mathrm{R}^{3}=\mathrm{Et} \mathbf{C} 2 ; \mathrm{R}^{1}=\mathrm{R}^{2}=\mathrm{H}, \mathrm{R}^{3}=\mathrm{i}-\mathrm{Bu} \mathrm{C} 3\right)$, while reaction of L2, $\mathbf{L} 3$ and $\mathbf{L} 4$ with solely $\mathrm{Me}_{3} \mathrm{Al}$ gave $\left[\left\{2-\mathrm{R}^{1}-7,7-\mathrm{Me}_{2}-8-\mathrm{R}^{2} \mathrm{C}_{9} \mathrm{H}_{6} \mathrm{~N}-8-\mathrm{O}\right\} \mathrm{AlMe}_{2}\right]_{2}\left(\mathrm{R}^{1}=\mathrm{Cl}, \mathrm{R}^{2}=\mathrm{H}\right.$ C4; $\left.\mathrm{R}^{1}=\mathrm{H}, \mathrm{R}^{2}=\mathrm{Me} \mathbf{C} 5 ; \mathrm{R}^{1}=\mathrm{Cl}, \mathrm{R}^{2}=\mathrm{Me} \mathbf{C 6}\right)$, in moderate to good yields. All six complexes have been characterized by ${ }^{1} \mathrm{H}$ and ${ }^{13} \mathrm{C}$ NMR spectroscopy as well as by elemental analysis. In addition, $\mathbf{C} \mathbf{1}$ and C4 have been the subject of single crystal X-ray diffraction studies. 


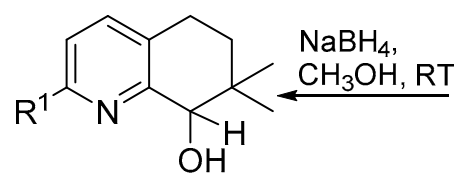

L1, $\mathrm{R}^{1}=\mathrm{H}$

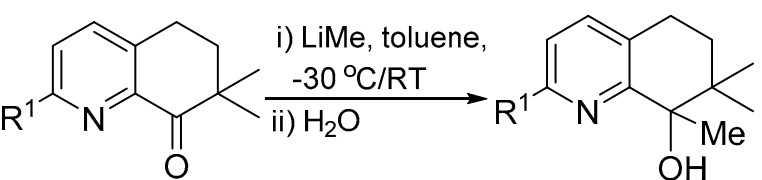

L3, $\mathrm{R}^{1}=\mathrm{H}$

L2, $\mathrm{R}^{1}=\mathrm{Cl}$

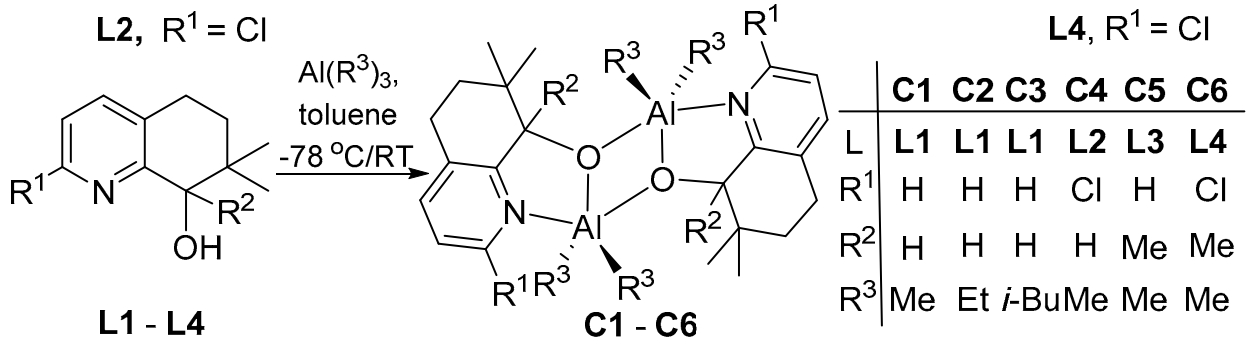

L4, $\mathrm{R}^{1}=\mathrm{Cl}$

Scheme 1. Synthetic routes to L1-L4 and aluminum complexes C1-C6.

Single crystals of $\mathbf{C} \mathbf{1}$ and $\mathbf{C 4}$ suitable for the X-ray determinations were grown by the slow diffusion of $n$-hexane into their toluene solutions at room temperature under a nitrogen atmosphere. Perspective views of the structures of $\mathbf{C} 1$ and $\mathbf{C} 4$ are shown in Figures 1 and 2; selected bond lengths and bond angles are collected in Table 2. Both $\mathbf{C} \mathbf{1}$ and $\mathbf{C} 4$ have been generated by symmetry and adopt similar arrangements and will be discussed together. Their structures consist of two aluminum centers that are each chelated by an $\mathrm{N}^{\wedge} \mathrm{O}$-bound 5,6-dihydro-7,7-dimethylquinolin-8-olate which also acts as a bridging ligand via $\mathrm{O} 1$. The five-coordinate geometry of each metal center is completed by two methyl ligands. The main difference between the structures relates to the substituent located on the 2-position of the $\mathrm{N}^{\wedge} \mathrm{O}$-chelate viz. $\mathrm{H}(\mathbf{C 1})$ and $\mathrm{Cl}(\mathbf{C} 4)$. Indeed, this dimeric arrangement is similar to that observed in the unsubstituted 5,6-dihydroquinolin-8-olates M (Chart 1) [37]. The Al-N bond length in C1 [2.122(13) A] is slightly shorter than that in C4 [2.194(3) A] likely reflecting the steric properties of the more bulky chloride substituent positioned at $\mathbf{C} 1$. There are no intermolecular contacts of note.

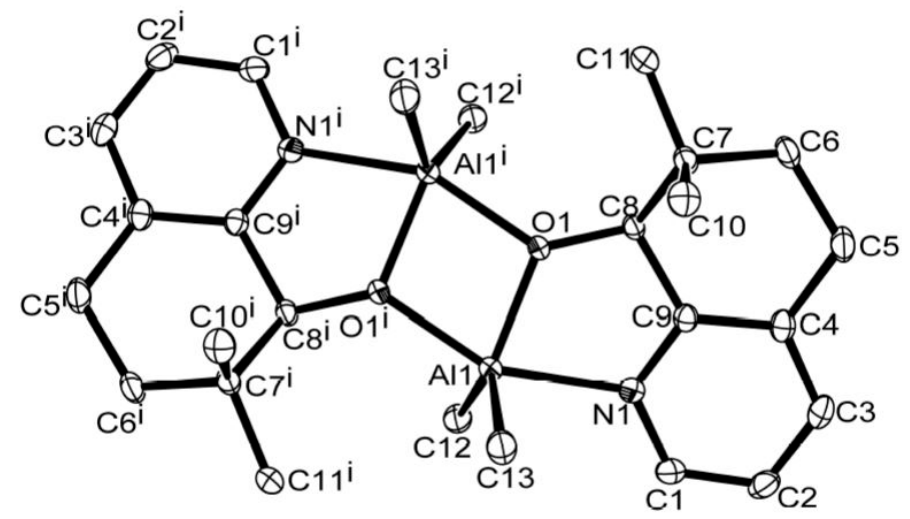

Figure 1. ORTEP representation of $\mathbf{C}$. Thermal ellipsoids are shown at 30\% probability while hydrogen atoms have been omitted for clarity. 


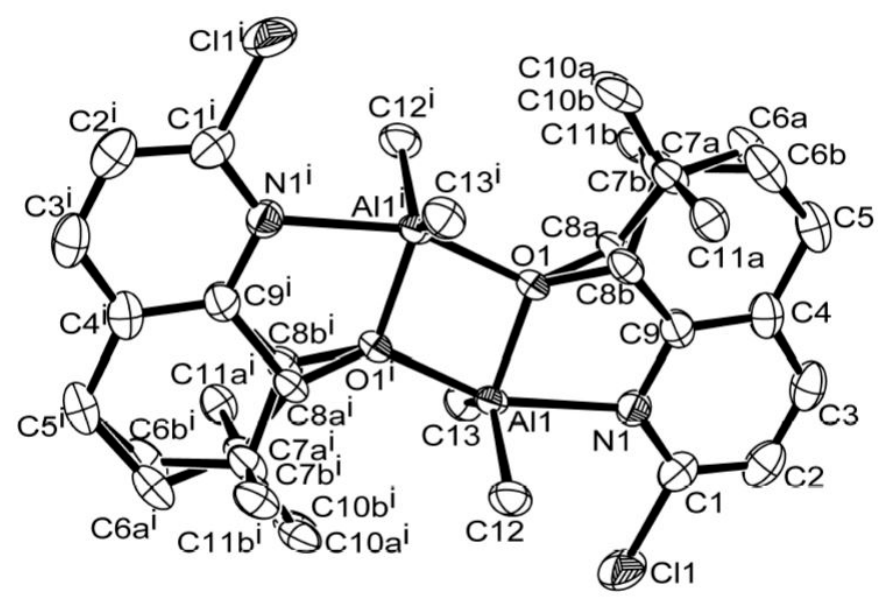

Figure 2. ORTEP representation of $\mathbf{C 4}$ showing the disorder in the saturated sections of the rings. Thermal ellipsoids are shown at $30 \%$ probability while hydrogen atoms have been omitted for clarity.

Table 2. Selected bond lengths $(\AA)$ and angles $\left(^{\circ}\right)$ for $\mathbf{C 1}$ and C4.

\begin{tabular}{ccc}
\hline & C1 & C4 \\
\hline & Bond lengths $(\AA)$ & \\
\hline $\mathrm{Al}(1)-\mathrm{N}(1)$ & $2.122(13)$ & $2.194(3)$ \\
$\mathrm{Al}(1)-\mathrm{O}(1)$ & $1.876(11)$ & $1.856(18)$ \\
$\mathrm{Al}(1)-\mathrm{O}(1 \mathrm{i})$ & $1.953(11)$ & $1.966(2)$ \\
$\mathrm{Al}(1)-\mathrm{C}(12)$ & $1.997(15)$ & $1.977(3)$ \\
$\mathrm{Al}(1)-\mathrm{C}(13)$ & $1.983(16)$ & $1.985(3)$ \\
$\mathrm{O}(1)-\mathrm{C}(8)$ & $1.427(15)$ & $1.398(5)$ \\
\hline $\mathrm{N}(1)-\mathrm{Al}(1)-\mathrm{O}(1)$ & Bond angles $\left(^{\circ}\right)$ & $78.73(9)$ \\
$\mathrm{N}(1)-\mathrm{Al}(1)-\mathrm{O}(1 \mathrm{i})$ & $79.14(5)$ & $158.62(8)$ \\
$\mathrm{Al}(1)-\mathrm{O}(1)-\mathrm{C}(8)$ & $154.49(5)$ & $121.8(3)$ \\
$\mathrm{Al}(1)-\mathrm{N}(1)-\mathrm{C}(9)$ & $121.91(8)$ & $79.93(18)$ \\
$\mathrm{O}(1)-\mathrm{Al}(1)-\mathrm{O}(1 \mathrm{i})$ & $113.13(9)$ & $134.10(13)$ \\
$\mathrm{C}(12)-\mathrm{Al}(1)-\mathrm{C}(13)$ & $77.25(5)$ & $123.79(7)$ \\
\hline
\end{tabular}

The atoms labeled with 'i' have been generated by symmetry.

The ${ }^{1} \mathrm{H}$ NMR spectra of $\mathbf{C 1}-\mathbf{C 6}$ reveal characteristic peaks for the corresponding 5,6-dihydro-7,7-dimethylquinolin-8-olate ligands with the inequivalent gem-dimethyl groups giving rise to two distinct signals. Likewise, the $\mathrm{Al}-\mathrm{CH}_{3}(\mathbf{C 1}, \mathbf{C} 4-\mathbf{C 6})$ or $\mathrm{Al}-\mathrm{CH}_{2}(\mathbf{C} 2, \mathrm{C} 3)$ resonances are seen, in most cases, as separate signals and appear most upfield in their spectra. Interestingly, the ${ }^{1} \mathrm{H}$ NMR spectrum of $\mathbf{C 1}$, recorded in three different deuterated solvents, indicates that the solvent polarity has some effect on the chemical shift of the resonances (Table 3). In an apolar solvent such as toluene- $d_{8}$ and benzene- $d_{6}$, each proton displays very similar chemical shifts. However, the shift in chloroform- $d$ is greatly affected, which is best exemplified by the most upfield $\mathrm{Cy}_{-} \mathrm{CH}_{3}$ signal which appears at $\delta 0.58$ in $\mathrm{C}_{6} \mathrm{D}_{6}$ while in $\mathrm{CDCl}_{3}$ at $\delta$ 0.82. In addition, the number of $\mathrm{Al}-\mathrm{CH}_{3}$ signals shows some variation with two peaks $(\delta-0.12,-0.26)$ in $\mathrm{C}_{6} \mathrm{D}_{6}$ and three peaks $(\delta-0.62,-0.66,-0.67)$ in $\mathrm{CDCl}_{3}$. It is uncertain as to the origin of these differences but it may be due to the ability of the more polar chloroform to interrupt the apparent monomeric/dimeric equilibrium in solution (vide infra). 
Table 3. The ${ }^{1} \mathrm{H}$ NMR chemical shifts for $\mathrm{C} 1$ recorded in different deuterated solvents.

\begin{tabular}{cccc}
\hline Proton Type & $\delta(\mathbf{p p m})$ in Toluene- $\boldsymbol{d}_{\mathbf{8}}$ & $\boldsymbol{\delta}(\mathbf{p p m})$ in Benzene- $\boldsymbol{d}_{\mathbf{6}}$ & $\boldsymbol{\delta}$ (ppm) in Chloroform- $\boldsymbol{d}$ \\
\hline $\mathrm{Py}-\mathrm{CH}$ & $7.44(1 \mathrm{H}), 6.63(1 \mathrm{H})$, & $7.29(1 \mathrm{H}), 6.43(1 \mathrm{H})$, & $8.24(1 \mathrm{H}), 7.79(1 \mathrm{H}), 7.50(1 \mathrm{H})$ \\
$\mathrm{Cy}-\mathrm{CH}$ & $6.43(1 \mathrm{H})$ & $6.23(1 \mathrm{H})$ & $4.92(1 \mathrm{H})$ \\
& $4.58(1 \mathrm{H})$ & $4.49(1 \mathrm{H})$ & $2.88(2 \mathrm{H}), 1.94-1.84$ \\
$\mathrm{Cy}-\mathrm{CH}_{2}$ & $2.04(2 \mathrm{H}), 1.20-1.11(2 \mathrm{H})$ & $1.86(2 \mathrm{H}), 1.08(1 \mathrm{H})$, & $(1 \mathrm{H}), 1.74-1.66(1 \mathrm{H})$ \\
$\mathrm{Cy}-\mathrm{CH}_{3}$ & $1.51(3 \mathrm{H}), 0.67(3 \mathrm{H})$ & $1.45(3 \mathrm{H}), 0.58(3 \mathrm{H})$ & $1.50(3 \mathrm{H}), 0.82(3 \mathrm{H})$ \\
$\mathrm{Al}-\mathrm{CH}_{3}$ & $-0.12(3 \mathrm{H}),-0.26(3 \mathrm{H})$ & $-0.12(3 \mathrm{H}),-0.25(3 \mathrm{H})$ & $-0.62,-0.66,-0.67$ \\
\hline
\end{tabular}

\subsection{Ring Opening Polymerization of $\varepsilon$-Caprolactone by $\mathbf{C 1 - C 6}$}

To explore the capacity of $\mathbf{C 1}-\mathbf{C} \mathbf{6}$ to serve as pro-initiators for the ring-opening polymerization of $\varepsilon-C L, C 1$ was firstly selected as the test system; the results of the catalytic evaluations are collected in Table 3. In the first instance, the study focused on establishing the optimal temperature by performing the polymerization runs between 25 and $110^{\circ} \mathrm{C}$. Typically, each run was conducted in toluene with one equivalent of $\mathrm{PhCH}_{2} \mathrm{OH}(\mathrm{BnOH})$ as activator and 250 equivalents of the monomer over a run time of $10 \mathrm{~min}$ (runs 1-7, Table 4). Below $40^{\circ} \mathrm{C}$, there was no monomer consumption after $10 \mathrm{~min}$. However, on raising the temperature from 60 to $90{ }^{\circ} \mathrm{C}$, the conversion gradually increased from $47 \%$ at $60^{\circ} \mathrm{C}$ to a maximum of $>99 \%$ at $90{ }^{\circ} \mathrm{C}$ (runs 4, 5, Table 4). Further increasing the temperature up to $110^{\circ} \mathrm{C}$ led to a steady decrease in the conversion. These findings contrast with observations seen for $\mathbf{M}$ (Chart 1$)$ in which only $20 \%$ conversion was observed at $80{ }^{\circ} \mathrm{C}$ and $95 \%$ at $90{ }^{\circ} \mathrm{C}$ [37].

Table 4. The ROP of $\varepsilon-C L$ using $C 1^{\text {a }}$.

\begin{tabular}{|c|c|c|c|c|c|c|c|c|c|}
\hline Run & $\varepsilon-C L: A l: B n O H$ & $\mathrm{~T}\left({ }^{\circ} \mathrm{C}\right)$ & $t(\min )$ & Solvent & $\begin{array}{l}\text { Conv. } \\
(\%)^{b}\end{array}$ & $\begin{array}{c}M_{\mathrm{n}} \\
\left(\times 10^{4}\right)^{\mathrm{c}}\end{array}$ & $\begin{array}{l}M_{\mathrm{n}}(\text { calc }) \\
\left(\times 10^{4}\right)^{\mathrm{d}}\end{array}$ & $\operatorname{PDI}^{c}$ & $\begin{array}{l}\text { TOF } \\
\left(h^{-1}\right)\end{array}$ \\
\hline 1 & 250:1:1 & 25 & 10 & Toluene & 0 & & & & \\
\hline 2 & $250: 1: 1$ & 40 & 10 & Toluene & 0 & & & & \\
\hline 3 & $250: 1: 1$ & 60 & 10 & Toluene & 47 & 1.11 & 1.35 & 1.27 & 705 \\
\hline 4 & $250: 1: 1$ & 80 & 10 & Toluene & 85 & 1.61 & 2.43 & 1.47 & 1275 \\
\hline 5 & 250:1:1 & 90 & 10 & Toluene & $>99$ & 1.89 & 2.83 & 1.61 & 1485 \\
\hline 6 & $250: 1: 1$ & 100 & 10 & Toluene & 95 & 1.84 & 2.72 & 1.72 & 1425 \\
\hline 7 & $250: 1: 1$ & 110 & 10 & Toluene & 92 & 1.84 & 2.63 & 1.76 & 1380 \\
\hline 8 & 250:1:1 & 90 & 3 & Toluene & 43 & 1.04 & 1.24 & 1.21 & 2150 \\
\hline 9 & 250:1:1 & 90 & 5 & Toluene & 63 & 1.35 & 1.81 & 1.41 & 1890 \\
\hline 10 & $250: 1: 1$ & 90 & 7 & Toluene & 86 & 1.18 & 2.46 & 1.43 & 1843 \\
\hline 11 & $250: 1: 0$ & 90 & 10 & Toluene & 0 & & & & \\
\hline 12 & 250:1:0 & 90 & 20 & Toluene & 0 & & & & \\
\hline 13 & 250:1:0 & 90 & 30 & Toluene & 15 & 0.27 & 0.43 & 1.15 & 75 \\
\hline 14 & $250: 1: 2$ & 90 & 10 & Toluene & 96 & 1.02 & 2.75 & 1.47 & 1440 \\
\hline 15 & 250:1:4 & 90 & 10 & Toluene & 85 & 0.61 & 2.43 & 1.37 & 1275 \\
\hline 16 & 250:1:5 & 90 & 10 & Toluene & 82 & 0.51 & 2.35 & 1.47 & 1230 \\
\hline 17 & $250: 1: 10$ & 90 & 10 & Toluene & 69 & 0.28 & 1.98 & 1.15 & 1035 \\
\hline 18 & 100:1:1 & 90 & 10 & Toluene & $>99$ & 0.75 & 2.83 & 1.40 & 594 \\
\hline 19 & 300:1:1 & 90 & 10 & Toluene & 93 & 1.58 & 2.66 & 1.47 & 1674 \\
\hline 20 & 400:1:1 & 90 & 10 & Toluene & 63 & 2.11 & 1.81 & 1.57 & 1512 \\
\hline 21 & 500:1:1 & 90 & 10 & Toluene & 51 & 2.23 & 1.46 & 1.49 & 1530 \\
\hline 22 & $750: 1: 1$ & 90 & 10 & Toluene & 23 & 2.51 & 0.67 & 1.35 & 1035 \\
\hline 23 & $250: 1: 1$ & 90 & 10 & Bulk & $>99$ & 0.81 & 2.83 & 1.64 & 1485 \\
\hline 24 & $250: 1: 1$ & - & 10 & Hexane & Trace & & & & \\
\hline 25 & $250: 1: 1$ & - & 10 & THF & Trace & & & & \\
\hline 26 & $250: 1: 1$ & - & 10 & $\mathrm{CH}_{2} \mathrm{Cl}_{2}$ & 9 & 0.40 & 0.27 & 2.03 & 135 \\
\hline $27^{\mathrm{e}}$ & $250: 1: 1$ & 90 & 30 & Bulk & trace & & & & \\
\hline $28^{\mathrm{e}}$ & $250: 1: 1$ & 60 & 30 & Hexane & 83 & 1.85 & 1.90 & & \\
\hline $29^{e}$ & $250: 1: 1$ & 60 & 30 & THF & 42 & 0.80 & 1.20 & & \\
\hline $30^{\mathrm{e}}$ & 250:1:1 & 35 & 30 & $\mathrm{CH}_{2} \mathrm{Cl}_{2}$ & 29 & 0.59 & 1.16 & & \\
\hline
\end{tabular}


With the $\varepsilon$-CL:Al:BnOH ratio fixed at 250:1:1 and the run temperature at $90{ }^{\circ} \mathrm{C}$, the influence of reaction time on the polymerization was explored (runs 5, 8-10, Table 4). Monitoring the polymerization by ${ }^{1} \mathrm{H}$ NMR spectroscopy at intervals of 3, 5, 7 and $10 \mathrm{~min}$ saw the conversion rise from $43 \%$ to approaching $100 \%$ while the molecular weight of polymer increased from $1.04 \times 10^{4}$ to $1.89 \times 10^{4} \mathrm{~g} / \mathrm{mol}$. By contrast, the TOF decreased with time implying gradual deactivation of the active species over longer run times. To investigate the importance of $\mathrm{BnOH}$ in the activation of $\mathbf{C} 1$, the polymerization runs were performed with and without benzyl alcohol over different run times but with the temperature maintained at $90^{\circ} \mathrm{C}$; the results are collected in Table 4 (runs 11-17). Without benzyl alcohol, $\mathbf{C} 1$ showed no activity after $20 \mathrm{~min}$ while after $30 \mathrm{~min}$ 15\% conversion was observed, highlighting the induction period needed when using $\mathbf{C} \mathbf{1}$ alone. This result contrasts with the virtually $100 \%$ conversion observed after $10 \mathrm{~min}$ using an equimolar ratio of $\mathbf{C} 1$ to $\mathrm{BnOH}$ (run 5, Table 4). On increasing the $\mathrm{BnOH} / \mathrm{Al}$ ratio from 1 to 10, the conversion gradually decreased from 99 to $69 \%$ (runs 5, 14-17, Table 4) while the $M_{\mathrm{n}}$ of the PCL lowered from $1.89 \times 10^{4}$ to $0.28 \times 10^{4} \mathrm{~g} / \mathrm{mol}$, suggesting the possible role of $\mathrm{BnOH}$ as a chain transfer reagent. An alternative explanation could be due to the likely intermediate ' $\mathbf{L 1} \mathrm{Al}\left(\mathrm{OCH}_{2} \mathrm{Ph}\right)_{2}$ ' undergoing disproportionation and dimerization to form an inactive species in a manner similar to that previously reported [41].

In order to gain some information on the structural features of the PCLs, all the samples obtained by employing different amounts of $\mathrm{BnOH}$ were analyzed by ${ }^{1} \mathrm{H}$ NMR spectroscopy and by MALDI-TOF mass spectrometry. In the case where no $\mathrm{BnOH}$ was employed, the MALDI-TOF spectrum indicated that the polymer was comprised of linear polymers capped by two different types of end-group ( $\alpha$ and $\beta$ in Figure 3). In $\alpha$ (the major species) the capping group is $\mathrm{CH}_{3}$ while in $\beta$ it is L1. These findings are supported by the ${ }^{1} \mathrm{H}$ NMR data that reveal aromatic signals characteristic of $\mathbf{L} \mathbf{1}$ in $\beta$ and a distinct singlet peak at $\delta 2.14$ that can be assigned to the acetyl end group $\left(\mathrm{CH}_{3} \mathrm{C}=\mathrm{O}\right)$ in $\alpha$ (Figure 4). Therefore, it would seem probable that this polymerization proceeded by a coordination-insertion mechanism in which the Al-Me and Al-L1 independently initiated the ring opening polymerization by pathways I and II (Scheme 2) [42-45].

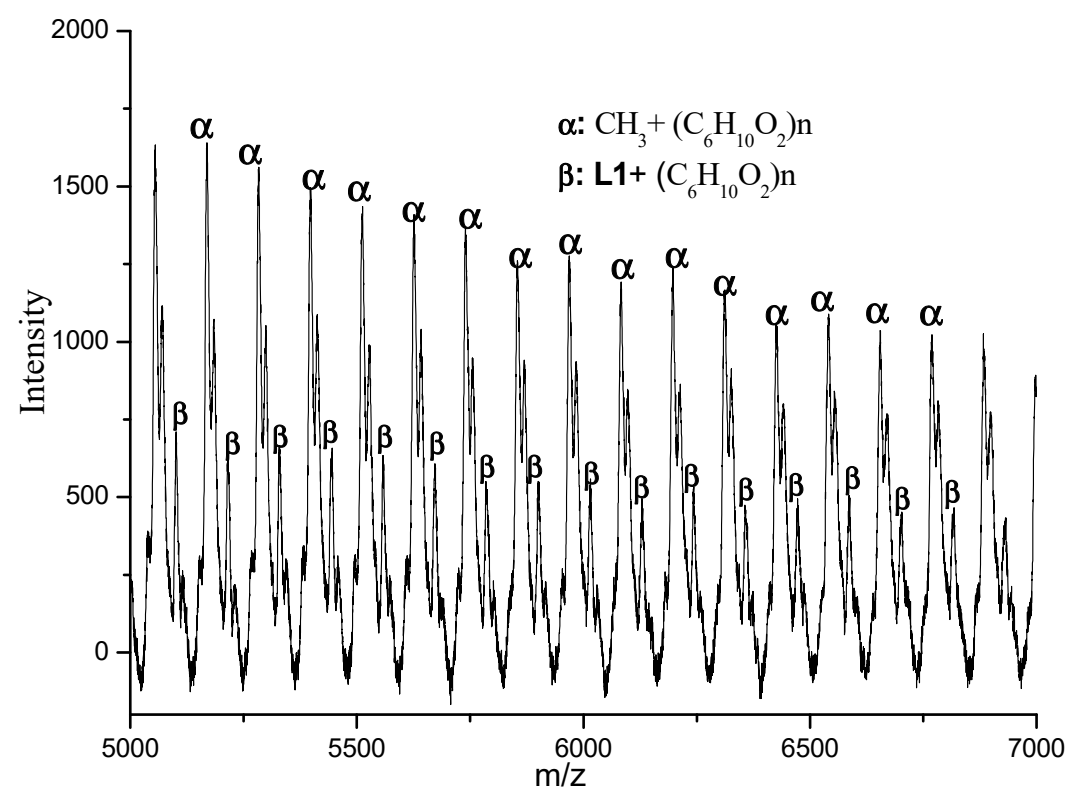

Figure 3. The MALDI-TOF mass spectrum of the PCL obtained using C1 (run 13, Table 3). 


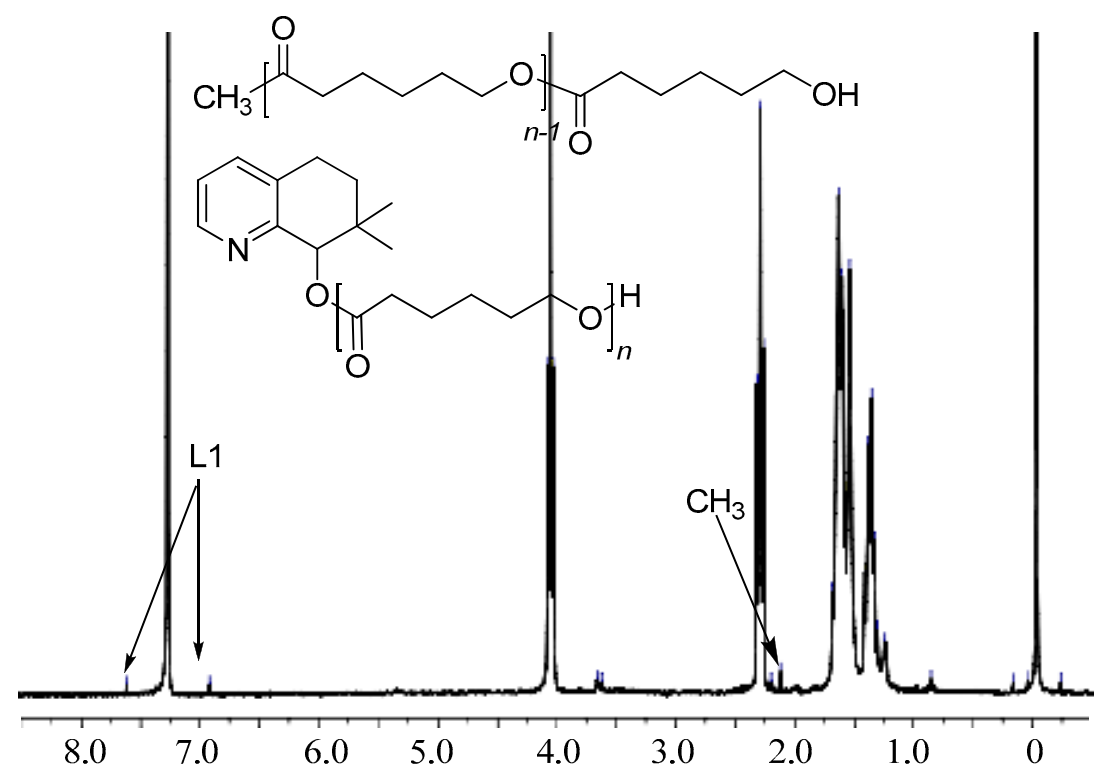

Figure 4. The ${ }^{1} \mathrm{H}$ NMR spectrum of the PCL obtained using $\mathbf{C 1}$ as initiator (run 13, Table 3).

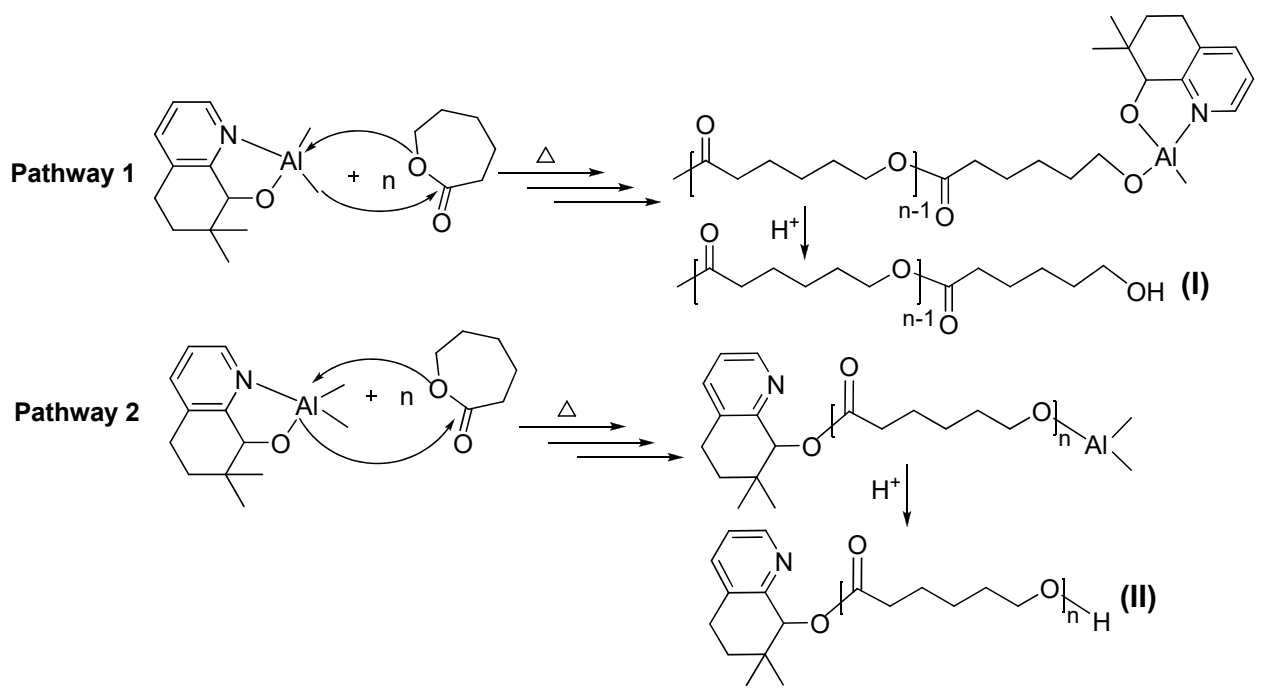

Scheme 2. Possible mechanistic pathways for the ring opening polymerization of $\varepsilon$-CL using C1.

On the other hand, when one equivalent or more of $\mathrm{BnOH}$ was employed in combination with $\mathbf{C 1}$, the ${ }^{1} \mathrm{H}$ NMR spectra of the PCLs showed only signals typical of $\mathrm{BnO}$ end groups (e.g., $\delta 5.0\left(\mathrm{OCH}_{2} \mathrm{Ph}\right)$ in Figure 5). This observation was corroborated by the MALDI-TOF spectrum that showed solely peaks corresponding to a linear chain polymer capped by a BnO group (Figure 6). Similarly, the polymers obtained by using larger amounts of $\mathrm{BnOH}$ possessed linear structures with $\mathrm{BnO}$ end groups, which were again confirmed by ${ }^{1} \mathrm{H}$ NMR spectroscopy and MALDI-TOF mass spectrometry (Figures S1-S4). As an additional feature of the spectra, it was evident that the more $\mathrm{BnOH}$ employed the lower the molecular weight of the polymer formed (runs 5, 14-17, Table 4). 


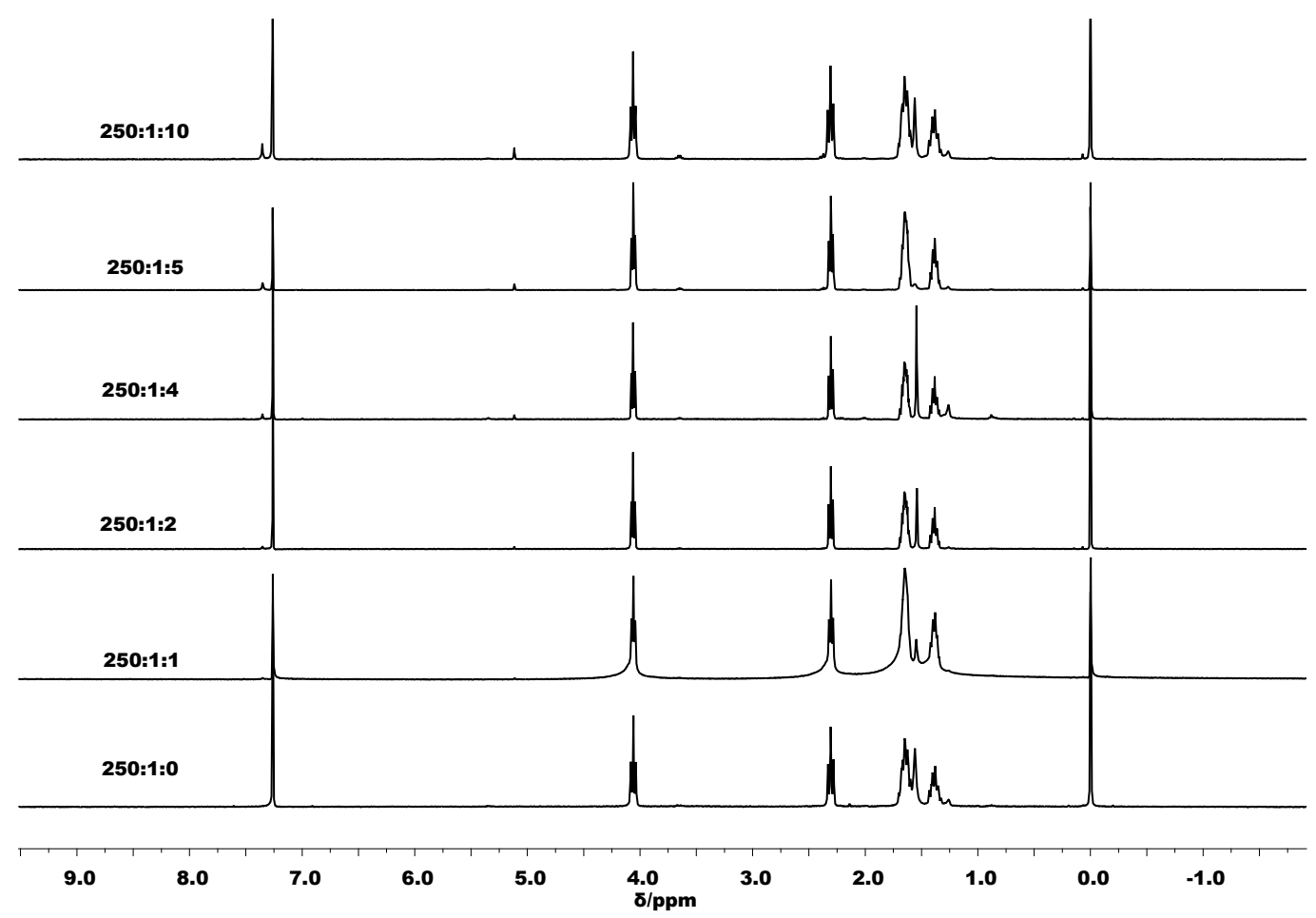

Figure 5. The ${ }^{1} \mathrm{H}$ NMR spectra of the PCL obtained using $\mathbf{C} \mathbf{1}$ with different amounts of $\mathrm{BnOH}$ (runs 5 , 13-17, Table 4).

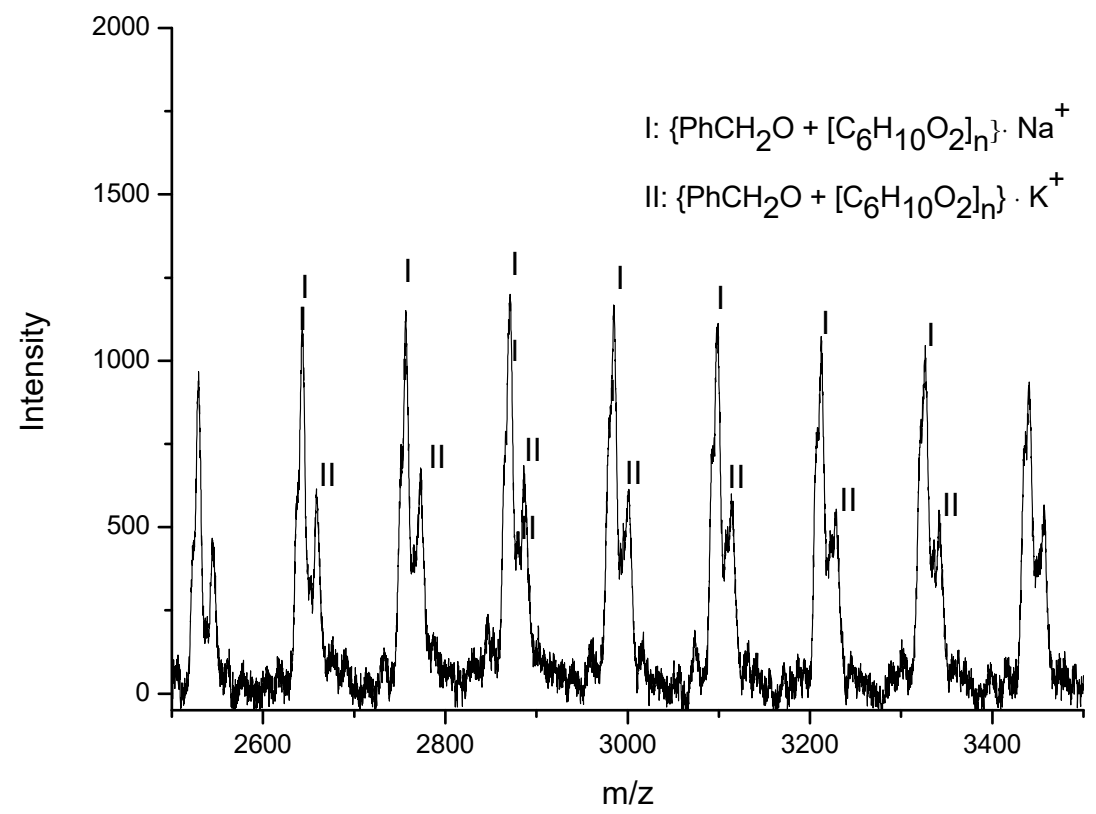

Figure 6. The MALDI-TOF mass spectrum of the PCL obtained using $\mathrm{C} 1 / \mathrm{BnOH}$ as initiator (run 5, Table 4).

With the amount of benzyl alcohol maintained at one molar equivalent, the effect of changing the amount of $\varepsilon$-CL on the performance of $\mathbf{C} 1$ was then examined. On increasing the molar ratio of $\varepsilon$-CL:Al from 100 to 300 , all the conversions were greater than $90 \%$ with the TOF showing an upward trend (runs $5,18,19$, Table 4). However, on further increasing the $\varepsilon-C L$ :Al ratio between 400 and 750, a dramatic decrease of both the conversion and TOF was evident (runs 20-22, Table 4). On the other hand, the molecular weight of the PCL was found to gradually increase from $0.75 \times 10^{4}$ to $2.51 \times 10^{4} \mathrm{~g} / \mathrm{mol}$ as 
the $\varepsilon$-CL:Al molar ratio was raised from 100 to 750 (runs 5, 18-22, Table 4), which can be explained by the faster rate of coordination and rate of propagation at higher monomer concentration.

The effect of solvent on the polymerizations using $\mathbf{C} 1$ was also studied. As a bulk polymerization was conducted at $90{ }^{\circ} \mathrm{C}$, almost $100 \%$ conversion was observed after $10 \mathrm{~min}$. The molecular weight was, however, much lower than that obtained in toluene (run 23, Table 4). When using n-hexane, dichloromethane or THF, the activity dramatically decreased and only trace amounts of polymer could be obtained (runs 24-26, Table 4). It is worthy to note that the above findings are significantly different to that found using analogue $\mathbf{M}$ (Chart 1). In that case, no activity was observed in bulk polymerization and moderate conversion was observed in other solvents [37]. One reason to account for these differences may be due to the improved solubility of $\mathbf{C} \mathbf{1}$ leading to more facile reactivity.

Based on the optimal conditions established for $\mathbf{C 1}$, the remaining five complexes, C2-C6, were also investigated for ROP; the results are collected in Table 4 alongside the data for $\mathbf{C 1}$. In terms of the steric properties of the aluminum- $\mathrm{R}^{3}$ group, replacing Me with an $i$-Bu group slightly decreased the conversion from 99 to $91 \%$ and the molecular weight from $1.89 \times 10^{4}$ to $1.21 \times 10^{4} \mathrm{~g} / \mathrm{mol}$ (runs 1-3, Table 5); the increased reactivity of an Al-Me over an Al-i-Bu towards $\mathrm{BnOH}$ may be a contributing factor. With a chloride present at the 2-position of the ligand, the conversion decreased rapidly as exemplified by $\mathbf{C} \mathbf{1}>\mathbf{C} 4$ and $\mathbf{C} 5>\mathbf{C} 6$. The reason for this observation is likely due to the electron withdrawing properties of a chloride resulting in decreased electron density at aluminum and in-turn slower reaction; steric factors could no doubt also be influential. In addition, the molecular weight of the PCLs generated by $\mathbf{C 5}$ and $\mathbf{C 6}\left(R^{2}=\mathrm{Me}\right)$ are slightly lower than that seen with $\mathbf{C} \mathbf{1}-\mathbf{C} 4\left(\mathrm{R}^{2}=\mathrm{H}\right)$, suggesting the detrimental effect of the $\mathrm{Cy}-\mathrm{CMeO}$ methyl group on chain propagation. Similar to $\mathbf{C} 1$, the MALDI-TOF mass spectra of the PCL generated by $\mathrm{C} 2-\mathrm{C} 5$ showed linear polymers capped with $\mathrm{BnO}$ groups as the unique polymer class. Likewise, the ${ }^{1} \mathrm{H}$ NMR spectra of PCLs generated by $\mathrm{C} 2-\mathrm{C} 5$ also clearly showed signals characteristic of a BnO group (Figures S5-S9). Indeed, these results are consistent with the polymerizations proceeding by 'coordination-insertion' route with ' $\mathrm{LAl}-\mathrm{OCH}_{2} \mathrm{Ph}^{\prime}$ presumed as the active species. Unexpectedly, the structural analysis of the PCL obtained by $\mathrm{C} 6 / \mathrm{BnOH}$ indicated that two polymer families were present, the major one being a linear structure capped with a $\mathrm{PhCH}_{2} \mathrm{O}$ group and the other a linear one capped with a methoxy group. These results suggest that the different $R^{1}$ and $R^{2}$ substituents have little effect on the polymer structure but have large effect on their reactivity.

Table 5. The ROP of $\varepsilon-C L$ promoted by $\mathrm{C} 1-\mathrm{C} 6 / \mathrm{BnOH}^{\mathrm{a}}$.

\begin{tabular}{|c|c|c|c|c|c|c|}
\hline Run & $\begin{array}{c}\text { Pro-Initiator } \\
\left(\mathbf{R}^{1}, \mathbf{R}^{2}, \mathbf{R}^{3}\right)\end{array}$ & Conv. $(\%)^{b}$ & $M_{\mathrm{n}}\left(\times 10^{4}\right)^{\mathrm{c}}$ & $M_{\mathrm{n}}($ calc. $)\left(\times 10^{4}\right)^{\mathrm{d}}$ & $\mathrm{PDI}^{\mathrm{c}}$ & TOF $\left(h^{-1}\right)$ \\
\hline 1 & $\mathrm{C1}(\mathrm{H}, \mathrm{H}, \mathrm{Me})$ & $>99$ & 1.89 & 2.83 & 1.61 & 1485 \\
\hline 2 & $\mathrm{C} 2(\mathrm{H}, \mathrm{H}, \mathrm{Et})$ & 95 & 1.35 & 2.72 & 1.32 & 1425 \\
\hline 3 & C3 $(\mathrm{H}, \mathrm{H}, \mathrm{i}-\mathrm{Bu})$ & 91 & 1.21 & 2.60 & 1.25 & 1365 \\
\hline 4 & $\mathrm{C} 4(\mathrm{Cl}, \mathrm{H}, \mathrm{Me})$ & 83 & 1.54 & 2.38 & 1.55 & 1245 \\
\hline 5 & $\mathrm{C} 5(\mathrm{H}, \mathrm{Me}, \mathrm{Me})$ & 97 & 0.73 & 2.78 & 1.30 & 1455 \\
\hline 6 & $\mathrm{C} 6(\mathrm{Cl}, \mathrm{Me}, \mathrm{Me})$ & 82 & 1.24 & 2.35 & 1.48 & 1230 \\
\hline
\end{tabular}

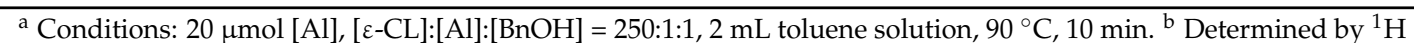

NMR spectroscopy. ${ }^{C}$ GPC data were recorded in THF vs. polystyrene standards, using a correcting factor of 0.56 [40]. ${ }^{\mathrm{d}} M_{\mathrm{n}}($ calcd $)=($ monomer $/$ initiator $) \times($ conversion $) \times 114+108($ molar mass of $\mathrm{BnOH})$.

\subsection{Mechanistic Analysis}

On account of the differences in polymerization behavior between $\mathbf{C 1 - C 6}$ and its unsubstituted counterpart $\mathbf{M}$ (Chart 1), the mode of activation was subject to a new ${ }^{1} \mathrm{H}$ and ${ }^{27} \mathrm{Al} \mathrm{NMR}$ spectroscopic investigation. In particular, toluene- $d_{8}$ solutions of $\mathbf{C 1}, \mathbf{C} 1 / \mathrm{BnOH}$ and $\mathbf{C 1} / \mathrm{BnOH}$ in the presence of 10 molar equivalents of $\varepsilon-C L$, were examined at various temperatures.

Firstly, the variable temperature ${ }^{1} \mathrm{H}$ NMR experiment was performed on $\mathbf{C} 1$ alone; the stacked spectra are shown in Figure 7. The data reveal the signals for the ligand show some modest shift to lower field with increasing temperature. On the other hand, two separate singlet peaks $(\delta-0.11,-0.24)$ 
for the Al-Me's at room temperature significantly shifted to lower field and became progressively broader on increasing the temperature. When the temperature reached $100{ }^{\circ} \mathrm{C}$, two peaks effectively collapsed at ca. $\delta 0$, which may be due to the onset of an exchange process at high temperature [46]. In the ${ }^{27} \mathrm{Al}$ NMR spectrum of $\mathbf{C} \mathbf{1}$, one broad peak around $\delta 176.83(\boldsymbol{a})$ was evident at room temperature. However, on raising the temperature from 25 to $100{ }^{\circ} \mathrm{C}$, a second more upfield peak $(\boldsymbol{b})(\delta 127.9)$ gradually grew in intensity (Figure 8 ). Based on these findings, we presume that $\mathbf{C} 1$ exists mainly as a dimeric species at low temperature which is partly transformed into a monomeric species at higher temperature in a manner similar to that previously reported [37].

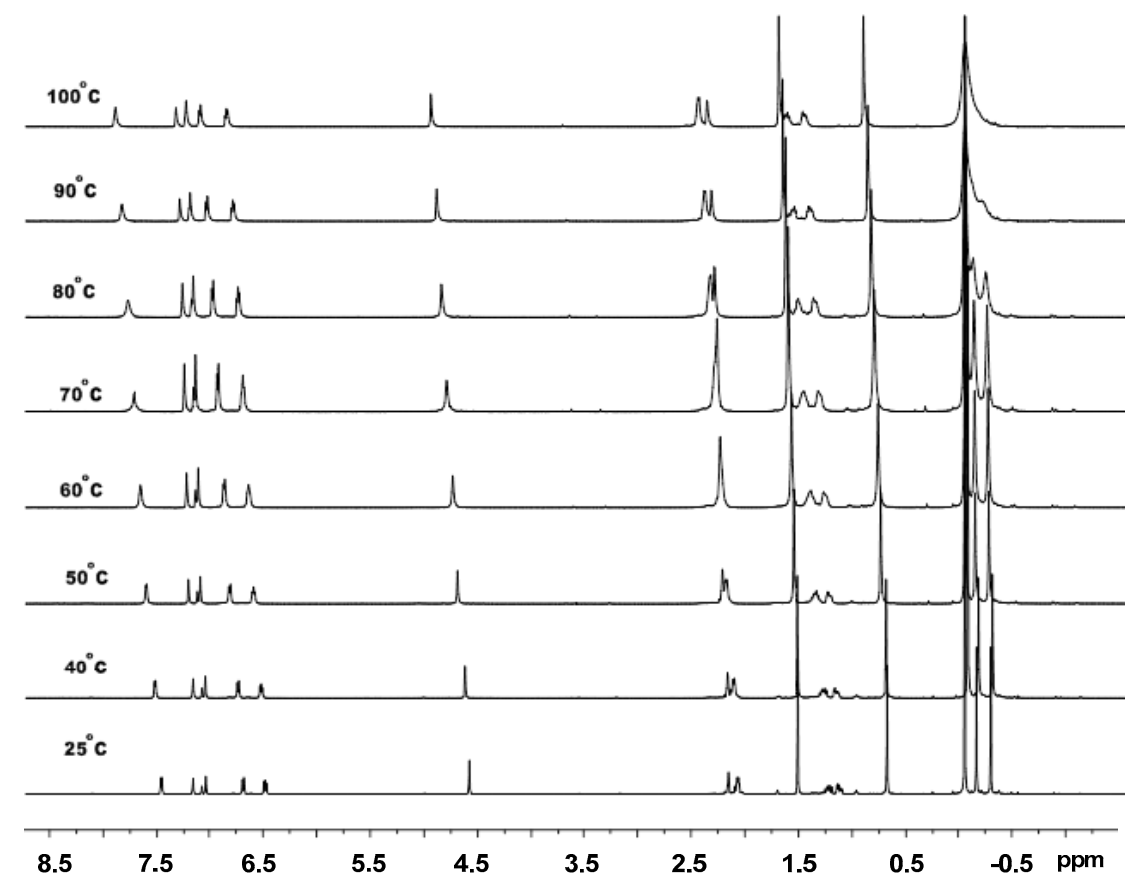

Figure 7. VT- ${ }^{1} \mathrm{H}$ NMR spectra of $\mathbf{C} 1$ recorded between 25 and $100{ }^{\circ} \mathrm{C}$ (in toluene- $d_{8}$ ).

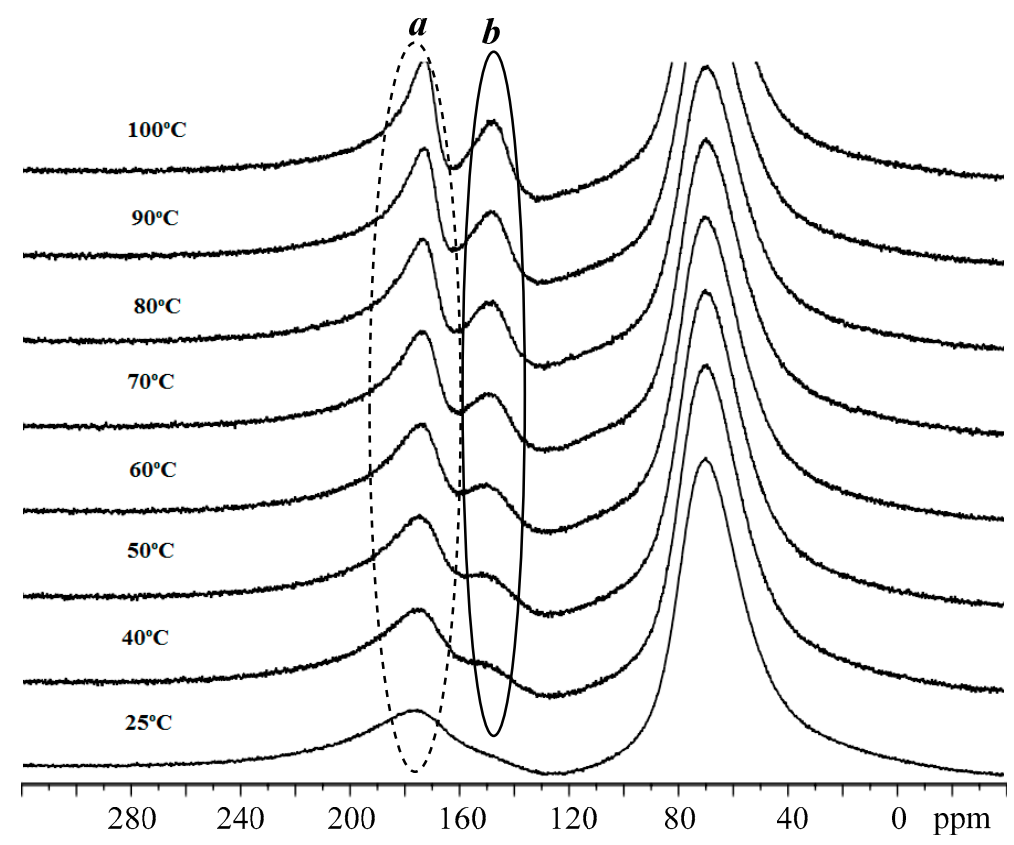

Figure 8. VT- ${ }^{27} \mathrm{Al}$ NMR spectra of $\mathbf{C} 1$ recorded between 25 and $100{ }^{\circ} \mathrm{C}$ (in toluene- $d_{8}$ ); the broad signal at $\delta 68.0$ derives from the aluminum alloy present in the NMR probe. 
We also monitored the variable temperature ${ }^{1} \mathrm{H}$ and ${ }^{27} \mathrm{Al} \mathrm{NMR}$ spectra of a 1:1 mixture of $\mathbf{C} \mathbf{1}$ and $\mathrm{BnOH}$; the corresponding spectra recorded between 25 and $100{ }^{\circ} \mathrm{C}$ are collected in Figures 9 and 10 . The ${ }^{1} \mathrm{H}$ NMR spectrum of $\mathbf{C} \mathbf{1} / \mathrm{BnOH}$ shows multiple upfield peaks for the Al-Me resonances at room temperature which converged into two sharp peaks on raising the temperature. By contrast, there were no major shifts of the downfield $\mathrm{H}_{\text {aryl }}$ protons over the temperature range. At room temperature, the coordinated benzyl alcohol methylene protons are inequivalent leading two mutually coupled doublets, this splitting pattern is maintained up to $70{ }^{\circ} \mathrm{C}$ above which the signals start to merge and broaden. Unexpectedly, the ratio of the $\mathrm{PhCH}_{2} \mathrm{O}: \mathrm{Al}-\mathrm{CH}_{3}$ protons is close to 2:6 at each temperature, which would appear to rule out a species of the type $\mathbf{L 1 A l}\left(\mathrm{OCH}_{2} \mathrm{Ph}\right)\left(\mathrm{CH}_{3}\right)$. In the ${ }^{27} \mathrm{Al} \mathrm{NMR}$ spectra of $\mathrm{C} 1 / \mathrm{BnOH}$, two broad peaks were observed in the temperature range 25 to $80{ }^{\circ} \mathrm{C}$. Further increasing the temperature to $90{ }^{\circ} \mathrm{C}$ led to apparent coalescence and then at $100{ }^{\circ} \mathrm{C}$ the formation of a single broad peak. By analogy with the NMR findings for $\mathbf{C} 1$ alone, we propose an equilibrating mixture of dimeric and monomeric species each coordinated by intact $\mathrm{BnOH}$ ligands that becomes time-averaged at high temperature.

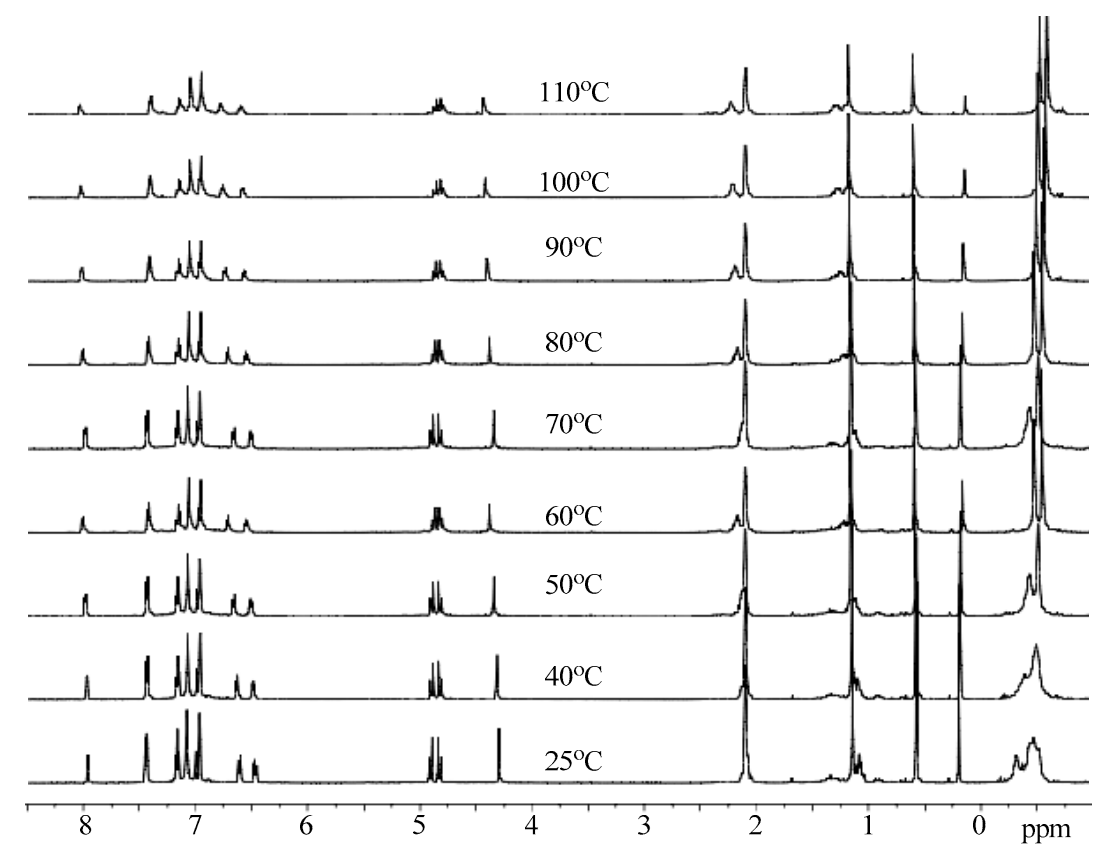

Figure 9. VT- ${ }^{1} \mathrm{H}$ NMR spectra of $\mathbf{C} 1 / \mathrm{BnOH}$ recorded between 25 and $110{ }^{\circ} \mathrm{C}$ (in toluene- $d_{8}$ ).

In order to gain information about the catalyst when in the presence of monomer, the ${ }^{1} \mathrm{H}$ and ${ }^{27} \mathrm{Al} \mathrm{NMR}$ spectra of $\mathbf{C 1} / \mathrm{BnOH}$ in the presence of 10 equivalents of $\varepsilon$-CL were also conducted over the 25 to $100{ }^{\circ} \mathrm{C}$ temperature range; the spectra are shown in Figure 11. In all the VT ${ }^{1} \mathrm{H}$ NMR spectra the $\mathrm{PhCH}_{2} \mathrm{O}$ resonance appeared as a singlet (around $\delta$ 5.0), which is indicative of a $\mathrm{PhCH}_{2} \mathrm{O}$ end group in PCL. Hence, it is apparent that the polymerization occurred rapidly when the monomer was mixed with $\mathrm{C} 1 / \mathrm{BnOH}$ even at room temperature. As the temperature was raised, the peaks for PCL $(\delta 3.94,1.52)$ gradually increased as the resonance for $\varepsilon$-CL $(\delta 3.58)$ reduced, in accord with the higher polymerization rate at higher temperature. On the other hand, the VT- ${ }^{27} \mathrm{Al} \mathrm{NMR} \mathrm{spectra} / \mathrm{BnOH} / 10$ $\varepsilon$-CL gave a single broad resonance at $\delta 69.0$ across the temperature range that we tentatively assign to the active species; unfortunately, this chemical shift also coincides with the probe signal. Nevertheless, related aluminum complexes containing bound $\alpha$-alkoxy esters, that have been considered as the active species in the ROP, have also shown resonances around $\delta 70$ in their ${ }^{27} \mathrm{Al} \mathrm{NMR}$ spectra [13]. 


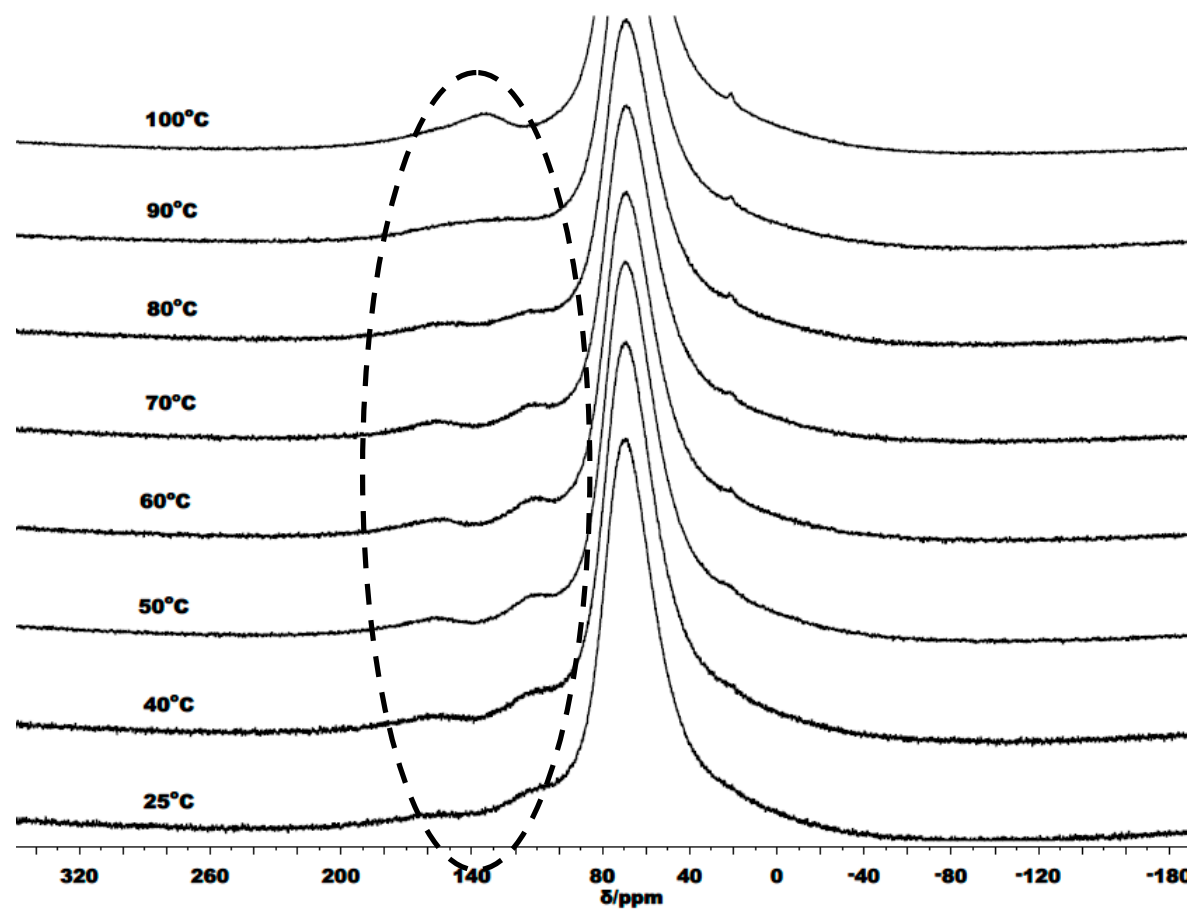

Figure 10. $\mathrm{VT}^{27} \mathrm{Al} \mathrm{NMR}$ spectra of $\mathrm{C} 1 / \mathrm{BnOH}$ recorded between 25 and $100{ }^{\circ} \mathrm{C}$ (in toluene- $d_{8}$ ); the broad signal at $\delta 68.0$ derives from the aluminum alloy present in the NMR probe.

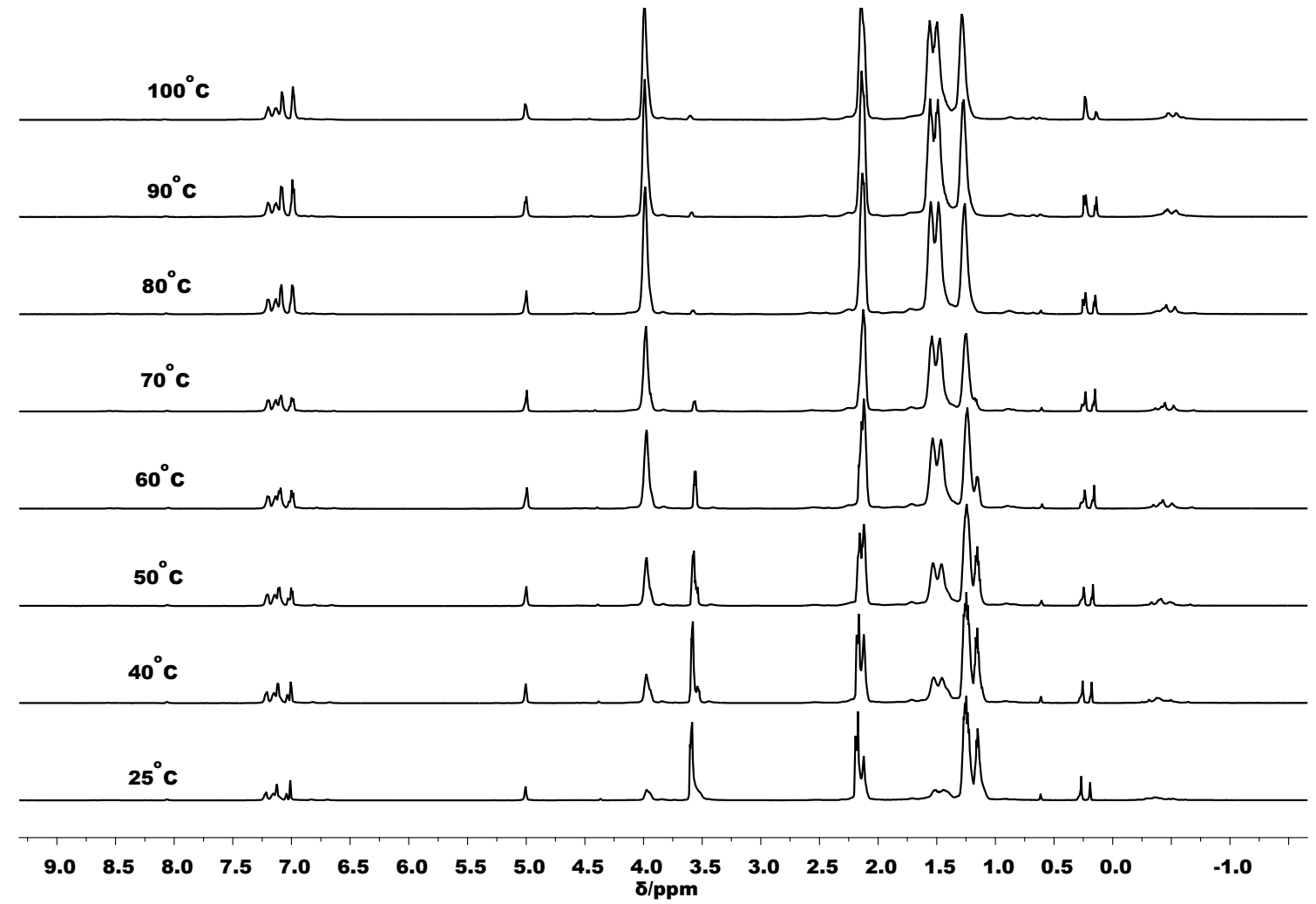

Figure 11. VT- ${ }^{1} \mathrm{H}$ NMR spectra of $\mathbf{C} 1 / \mathrm{BnOH} / 10 \varepsilon-\mathrm{CL}$ recorded at temperatures between 25 and $100{ }^{\circ} \mathrm{C}$ (in toluene- $d_{8}$ ). 


\section{Conclusions}

A series of soluble aluminum 5,6-dihydro-7,7-dimethylquinolin-8-olates, C1-C6, have been successfully prepared and fully characterized. The molecular structures of $\mathbf{C} 1$ and $\mathbf{C} 4$ indicate that they adopt dimeric forms in the solid state. In the absence of $\mathrm{BnOH}, \mathbf{C 1}$ showed only low catalytic efficiency for the ROP of $\varepsilon$-CL with the spectroscopic and spectrometric data for the resulting polymer in agreement with a linear structure capped with either a methyl or a $\mathbf{L 1}$ group. By contrast, in the presence of $\mathrm{BnOH}, \mathrm{C} 1$ exhibited excellent efficiency for the ROP of $\varepsilon$-CL with essentially $100 \%$ conversion in only $10 \mathrm{~min}$ at $90^{\circ} \mathrm{C}$. The chloro-substituted complexes $\mathbf{C} 4$ and $\mathbf{C} 6$ showed lower activity than that seen with C1-C3 and C5, highlighting the importance of both electronic and steric factors on initiator performance. All polymers displayed linear structures that were capped with $\mathrm{PhCH}_{2} \mathrm{O}$ groups, which was confirmed by ${ }^{1} \mathrm{H}$ NMR spectroscopy and by MALDI-TOF mass spectrometry. The solution state properties of $\mathbf{C} 1$ and $\mathbf{C 1} / \mathrm{BnOH}$ over various temperatures have been investigated by multinuclear NMR spectroscopy and highlight the importance of both monomeric and dimeric species. In the presence of 10 molar equivalents of monomer, the active initiator generated from $\mathrm{C} 1 / \mathrm{BnOH}$, could be monitored by ${ }^{27} \mathrm{Al}$ NMR spectroscopy while the chain propagation by ${ }^{1} \mathrm{H}$ NMR spectroscopy.

Supplementary Materials: The following are available online at http:/ /www.mdpi.com/2073-4360/10/7/764/s1, Figures S1-S4: The ${ }^{1} \mathrm{H}$ NMR and MALDI-TOF spectrum of PCL obtained by $\mathbf{C} 1$ with different amount $\mathrm{BnOH}$, Figures S5-S9: The MALDI-TOF spectrum of PCL obtained by $\mathbf{C 2}-\mathbf{C 6} / \mathbf{B n O H}$.

Author Contributions: W.-H.S. and W.Z. conceived and designed the experiments; Q.Z. performed the experimental work and the analysis of structural data; T.L. took the single crystal determination. W.Z., W.-H.S. contributed regents/materials/analysis. W.Z., W.-H.S. and G.A.S. wrote the paper.

Acknowledgments: This research was funded by National Natural Science Foundation of China (Nos 51473170, 51273202 and U1362204). G.A.S. thanks the Chinese Academy of Sciences for a President's International Fellowship for Visiting Scientists.

Conflicts of Interest: The authors declare no conflict of interest.

\section{References}

1. Reddy, M.M.; Vivekanandhan, S.; Misra, M.; Bhatia, S.K.; Mohanty, A.K. Biobased plastics and bionanocomposites: Current status and future opportunities. Prog. Polym. Sci. 2013, 38, 1653-1689. [CrossRef]

2. Singhvi, M.; Gokhale, D. Biomass to biodegradable polymer (PLA). RSC Adv. 2013, 3, 13558-13568. [CrossRef]

3. Rhim, J.-W.; Park, H.-M.; Ha, C.-S. Bio-nanocomposites for food packaging applications. Prog. Polym. Sci. 2013, 38, 1629-1652. [CrossRef]

4. Armentano, I.; Bitinis, N.; Fortunati, E.; Mattioli, S.; Rescignano, N.; Verdejo, R.; Lopez-Manchado, M.A.; Kenny, J.M. Multifunctional nanostructured PLA materials for packaging and tissue engineering. Prog. Polym. Sci. 2013, 38, 1720-1747. [CrossRef]

5. Terzopoulou, Z.; Baciu, D.; Gounari, E.; Steriotis, T.; Charalambopoulou, G.; Bikiaris, D. Biocompatible Nanobioglass Reinforced Poly( $\varepsilon$-Caprolactone) Composites Synthesized via In Situ Ring Opening Polymerization. Polymers 2018, 10, 381. [CrossRef]

6. Wei, Y.; Wang, S.; Zhou, S. Aluminum alkyl complexes: Synthesis, structure, and application in ROP of cyclic esters. Dalton Trans. 2016, 45, 4471-4485. [CrossRef] [PubMed]

7. Arbaoui, A.; Redshaw, C. Metal catalysts for $\varepsilon$-caprolactone polymerization. Polym. Chem. 2010, 1, 801-826. [CrossRef]

8. Spassky, N.; Wisniewski, M.; Pluta, C.; Le Borgne, A. Highly stereoelective polymerization of rac-(D,L)-lactide with a chiral Schiff's base/aluminium alkoxide initiator. Macromol. Chem. Phys. 1996, 197, 2627-2637. [CrossRef]

9. Ovitt, T.M.; Coates, G.W. Stereoselective ring-opening polymerization of meso-Lactide: Synthesis of syndiotactic poly(lactic acid). J. Am. Chem. Soc. 1999, 121, 4072-4073. [CrossRef]

10. Tang, Z.H.; Chen, X.S.; Pang, X.; Yang, Y.K.; Zhang, X.F.; Jing, X.B. Stereoselective polymerization of rac-lactide using a monoethylaluminum schiff base complex. Biomacromolecules 2004, 5, 965-970. [CrossRef] [PubMed] 
11. Radano, C.P.; Baker, G.L.; Smith, M.R. Stereoselective polymerization of a racemic monomer with a racemic catalyst: Direct preparation of the polylactic acid stereocomplex from racemic lactide. J. Am. Chem. Soc. 2000, 122, 1552-1553. [CrossRef]

12. Zhang, W.; Wang, Y.; Sun, W.-H.; Wang, L.; Redshaw, C. Dimethylaluminium aldiminophenolates: Synthesis, characterization and ring-opening polymerization behavior towards lactides. Dalton Trans. 2012, 41, 11587-11596. [CrossRef] [PubMed]

13. Kan, C.; Ge, J.L.; Ma, H.Y. Aluminum methyl, alkoxide and $\alpha$-alkoxy ester complexes supported by 6,6'-dimethylbiphenyl-bridged salen ligands: Synthesis, characterization and catalysis for rac-lactide polymerization. Dalton Trans. 2016, 45, 6682-6695. [CrossRef] [PubMed]

14. Iwasa, N.; Fujiki, M.; Nomura, K. Ring-opening polymerization of various cyclic esters by $\mathrm{Al}$ complex catalysts containing a series of phenoxy-imine ligands: Effect of the imino substituents for the catalytic activity. J. Mol. Catal. A Chem. 2008, 292, 67-75. [CrossRef]

15. Iwasa, N.; Katao, S.; Liu, J.Y.; Fujiki, M.; Furukawa, Y.; Nomura, K. Notable effect of fluoro substituents in the imino group in ring-opening polymerization of $\varepsilon$-caprolactone by $\mathrm{Al}$ complexes containing phenoxyimine ligands. Organometallics 2009, 28, 2179-2187. [CrossRef]

16. Meduri, A.; Fuoco, T.; Lamberti, M.; Pellecchia, C.; Pappalardo, D. Versatile copolymerization of glycolide and rac-lactide by dimethyl(salicylaldiminato)aluminum compounds. Macromolecules 2014, 47, 534-543. [CrossRef]

17. García-Valle, F.M.; Tabernero, V.; Tomas, C.; Mosquera, M.E.G.; Cano, J.; Milione, S. Biodegradable PHB from rac- $\beta$ butyrolactone: Highly controlled ROP mediated by a pentacoordinated aluminum complex. Organometallics 2018, 37, 837-840. [CrossRef]

18. Shi, T.; Luo, W.L.; Liu, S.F.; Li, Z.B. Controlled random copolymerization of rac-lactide and caprolactone by well-designed phenoxyimine Al complexes. J. Polym. Sci. Part A Polym. Chem. 2018, 56, 611-617. [CrossRef]

19. Shi, T.; Zheng, Q.; Zuo, W.; Liu, S.; Li, Z. Bimetallic aluminum complexes supported by bis(salicylaldimine) ligand: Synthesis, characterization and ring-opening polymerization of lactide. Chin. J. Polym. Sci. 2018, 36, 149-156. [CrossRef]

20. Maisse-François, A.; Azora, L.; Schmitta, A.-L.; Coquela, A.; Brelotc, L.; Welter, R.; Bellemin-Laponnaz, S.; Dagorne, S. Structural diversity and versatility for organoaluminum complexes supported by mono- and di-anionic aminophenolate bidentate ligands. J. Organomet. Chem. 2012, 696, 4248-4256. [CrossRef]

21. Wang, Y.; Ma, H.Y. Aluminum complexes of bidentate phenoxy-amine ligands: Synthesis, characterization and catalysis in ring-opening polymerization of cyclic esters. J. Organomet. Chem. 2013, 731, 23-28. [CrossRef]

22. Chen, L.; Li, W.; Yuan, D.; Zhang, Y.; Shen, Q.; Yao, Y. Syntheses of Mononuclear and Dinuclear Aluminum Complexes Stabilized by Phenolato Ligands and Their Applications in the Polymerization of $\varepsilon$ Caprolactone: A Comparative Study. Inorg. Chem. 2015, 54, 4699-4708. [CrossRef] [PubMed]

23. Li, W.; Ouyang, H.; Chen, L.J.; Yuan, D.; Zhang, Y.; Yao, Y. A Comparative Study on Dinuclear and Mononuclear Aluminum Methyl Complexes Bearing Piperidyl-Phenolato Ligands in ROP of Epoxides. Inorg. Chem. 2016, 55, 6520-6524. [CrossRef] [PubMed]

24. Roymuhury, S.K.; Chakraborty, D.; Ramkumar, V. Aluminium complexes bearing N,O-aminophenol ligands as efficient catalysts for the ring opening polymerization of lactide. Eur. Polym. J. 2015, 70, 203-214. [CrossRef]

25. Zhang, W.; Wang, Y.; Wang, L.; Redshaw, C.; Sun, W.-H. Dialkylaluminium 2-imidazolylphenolates: Synthesis, characterization and ring-opening polymerization behavior towards lactides. J. Organomet. Chem. 2014, 750, 65-73. [CrossRef]

26. Sumrit, P.; Chuawong, P.; Nanok, T.; Duangthongyou, T.; Hormnirun, P. Aluminum complexes containing salicylbenzoxazole ligands and their application in the ring-opening polymerization of rac-lactide and $\varepsilon$-caprolactone. Dalton Trans. 2016, 45, 9250-9266. [CrossRef] [PubMed]

27. Liu, Y.; Dong, W.S.; Liu, J.Y.; Li, Y.S. Living ring-opening homo- and copolymerisation of $\varepsilon$-caprolactone and L-lactide by cyclic $\beta$-ketiminato aluminium complexes. Dalton Trans. 2014, 43, 2244-2251. [CrossRef] [PubMed]

28. Bouyahyi, M.; Roisnel, T.; Carpentier, J.-F. Aluminum Complexes of Bidentate Fluorinated Alkoxy-Imino Ligands: Syntheses, Structures, and Use in Ring-Opening Polymerization of Cyclic Esters. Organometallics 2012, 31, 1458-1466. [CrossRef] 
29. Liang, L.-C.; Chen, F.-Y.; Huang, M.-H.; Cheng, L.-C.; Li, C.-W.; Lee, H.M. Aluminium complexes of bidentate $\mathrm{N}, \mathrm{O}$ - and $\mathrm{N}, \mathrm{N}$-ligands derived from oxidative functionalization of amido phosphines: Synthesis, structure and reactivity. Dalton Trans. 2010, 39, 9941-9951. [CrossRef] [PubMed]

30. Lee, C.-L.; Lin, Y.-F.; Jiang, M.-T.; Lu, W.-Y.; Vandavasi, J.K.; Wang, L.-F.; Lai, Y.-C.; Chiang, M.Y.; Chen, H.-Y. Improvement in aluminum complexes bearing schiff bases in ring opening polymerization of $\varepsilon$ caprolactone: A five-membered-ring system. Organometallics 2017, 36, 1936-1945. [CrossRef]

31. Francis, J.A.; Bott, S.G.; Barron, A.R. Aluminium compounds containing bidentate ligands: Chelate ring size and rigid conformation effects. J. Chem. Soc. Dalton Trans. 1998, 3305-3310. [CrossRef]

32. Francis, J.A.; Bott, S.G.; Barron, A.R. Sterically crowded aryloxides of aluminum: Intramolecular coordination of bidentate ligands. J. Organomet. Chem. 2000, 597, 29-37. [CrossRef]

33. Galvez-Ruiz, J.C.; Noth, H.; Flores-Parra, A. Organometallic Aluminum Compounds Derived from 2-(1,3,5-Dithiazinan-5-yl)ethanol Ligands. Inorg. Chem. 2003, 42, 7569-7578. [CrossRef] [PubMed]

34. Kim, S.H.; Han, S.Y.; Kim, J.H.; Kang, Y.Y.; Lee, J.; Kim, Y. Monomeric or dimeric aluminum complexes as catalysts for cycloaddition between $\mathrm{CO}_{2}$ and epoxides. Eur. J. Inorg. Chem. 2015, 2323-2329. [CrossRef]

35. Li, M.; Chen, M.; Chen, C.L. Ring-opening polymerization of rac-lactide using anilinotroponebased aluminum complexes-sidearm effect on the catalysis. Polymer 2015, 64, 234-239. [CrossRef]

36. Sun, W.-H.; Shen, M.; Zhang, W.J.; Huang, W.; Liu, S.F.; Redshaw, C. Methylaluminium 8-quinolinolates: Synthesis, characterization and use in ring-opening polymerization (ROP) of $\varepsilon$-caprolactone. Dalton Trans. 2011, 40, 2645-2653. [CrossRef] [PubMed]

37. Zhang, Q.; Zhang, W.; Rajendran, N.; Liang, T.; Sun, W.-H. Thermo-enhanced ring-opening polymerization of $\varepsilon$-caprolactone: The synthesis, characterization, and catalytic behavior of aluminum hydroquinolin-8-olates. Dalton Trans. 2017, 46, 7833-7843. [CrossRef] [PubMed]

38. Sheldrick, G.M. SHELXT-Integrated space-group and crystal-structure determination. Acta Crystallogr. Sect. A Found. Adv. 2015, 71, 3-8. [CrossRef] [PubMed]

39. Sheldrick, G.M. Crystal structure refinement with SHELXL. Acta Crystallogr. Sect. C Struct. Chem. 2015, 71, 3-8. [CrossRef] [PubMed]

40. Save, M.; Schappacher, M.; Soum, A. Controlled Ring-Opening Polymerization of Lactones and Lactides Initiated by Lanthanum Isopropoxide, General Aspects and Kinetics. Macromol. Chem. Phys. 2002, 203, 889-899. [CrossRef]

41. Tseng, H.-C.; Chiang, M.Y.; Lu, W.-Y.; Chen, Y.-J.; Lian, C.-J.; Chen, Y.-H.; Tsai, H.-Y.; Lai, Y.-C.; Chen, H.-Y. A closer look at $\varepsilon$-caprolactone polymerization catalyzed by alkyl aluminum complexes: The effect of induction period on overall catalytic activity. Dalton Trans. 2015, 44, 11763-11773. [CrossRef] [PubMed]

42. Chakraborty, D.; Chen, E.Y.-X. Neutral, three-coordinate, chelating diamide aluminum complexes: Catalysts/initiators for synthesis of telechelic oligomers and high polymers. Organometallics 2002, 21, 1438-1442. [CrossRef]

43. Yu, R.-C.; Hung, C.-H.; Huang, J.-H.; Lee, H.-Y.; Chen, J.-T. Four- and five-Coordinate aluminum ketiminate complexes: Synthesis, characterization, and ring-opening polymerization. Inorg. Chem. 2002, 41, 6450-6455. [CrossRef] [PubMed]

44. Wei, Y.; Wang, S.; Zhu, X.; Zhou, S.; Mu, X.; Huang, Z.; Hong, D. Aluminum Complexes Bearing N-Protected 2-Amino- or 2-Imino-Functionalized Pyrrolyl Ligands: Synthesis, Structure, and Catalysis for Preparation of Pyrrolyl-End-Functionalized Polyesters. Organometallics 2016, 35, 2621-2629. [CrossRef]

45. Li, H.; Debuigne, A.; Jrome, R.; Lecomte, P. Synthesis of Macrocyclic poly( $\varepsilon$-caprolactone) by intramolecular cross-Linking of unsaturated end groups of chains precyclic by the initiation. Angew. Chem. Int. Ed. 2006, 45, 2264-2267. [CrossRef] [PubMed]

46. Kumar, R.; Sierra, M.L.; Oliver, J.P. Synthesis and Spectroscopic Studies of Alkylaluminum Alkoxides Derived from Optically Active Alcohols. Crystal and Molecular Structure of Monomeric Dimethylaluminum (2S,3R)-(+)-4-(Dimethylamino)-1,2-diphenyl-3-methyl-2-butanoxide. Organometallics 1994, 13, 4285-4293. [CrossRef]

(C) 2018 by the authors. Licensee MDPI, Basel, Switzerland. This article is an open access article distributed under the terms and conditions of the Creative Commons Attribution (CC BY) license (http:/ / creativecommons.org/licenses/by/4.0/). 